University of Louisville

ThinkIR: The University of Louisville's Institutional Repository

Electronic Theses and Dissertations

$12-2011$

\title{
Avoiding, pretending, and querying : three combinatorial problems.
}

Adam S. Jobson

University of Louisville

Follow this and additional works at: https://ir.library.louisville.edu/etd

\section{Recommended Citation}

Jobson, Adam S., "Avoiding, pretending, and querying : three combinatorial problems." (2011). Electronic Theses and Dissertations. Paper 693.

https://doi.org/10.18297/etd/693

This Doctoral Dissertation is brought to you for free and open access by ThinkIR: The University of Louisville's Institutional Repository. It has been accepted for inclusion in Electronic Theses and Dissertations by an authorized administrator of ThinkIR: The University of Louisville's Institutional Repository. This title appears here courtesy of the author, who has retained all other copyrights. For more information, please contact thinkir@louisville.edu. 


\title{
AVOIDING, PRETENDING, AND QUERYING: THREE COMBINATORIAL PROBLEMS
}

\author{
By \\ Adam S. Jobson \\ B.S., University of Louisville, 2004 \\ M.A., University of Louisville, 2006

\begin{abstract}
A Dissertation
Submitted to the Faculty of the

College of Arts and Sciences of the University of Louisville in Partial Fulfillment of the Requirements

for the Degree of
\end{abstract}

Doctor of Philosophy

Department of Mathematics

University of Louisville

Louisville, Kentucky

December 2011 


\section{Copyright 2011 by Adam S. Jobson}

\section{(c) $\mathrm{BY}-\mathrm{SA}$}

This work is licensed under the Creative Commons Attribution-ShareAlike 3.0 Unported License. To view a copy of this license, visit http://creativecommons .org/licenses/by-sa/3.0/ or send a letter to Creative Commons, 444 Castro Street, Suite 900, Mountain View, California, 94041, USA. 


\section{AVOIDING, PRETENDING, AND QUERYING: THREE COMBINATORIAL PROBLEMS}

\section{By}

Adam S. Jobson

B.S., University of Louisville, 2004

M.A., University of Louisville, 2006

A Dissertation Approved on

18 November 2011

By the following Dissertation Committee:

Andre Kezdy, Dissertation Director

Manav Das 
To my parents. 


\section{ACKNOWLEDGEMENTS}

I wish to thank my advisor, Professor André Kézdy, for his patience and encouragement. I have learned more from him than I can ever repay.

To my family, for their unwavering belief in me.

To Marshall Lagani, Ben Allgeier, Tim Brauch, and Lesley Wellington Wiglesworth, for countless hours of collaboration, both in mathematics and in life.

To Professors Udayan Darji and Manav Das, for introducing me to higher mathematics and encouraging me to pursue graduate work.

To Professor Thomas Riedel and everybody in the University of Louisville Department of Mathematics, for their years of support.

To Jim Conrey, for introducing me to programming. I cannot imagine where I would be without it.

To Donald Knuth for creating TEX, Leslie Lamport for creating $\mathrm{LT}_{\mathrm{E}} \mathrm{X}$, Till Tantau for creating TikZ, and the thousands of others who have contributed to these projects. Mathematics is even more beautiful thanks to their efforts. 


\begin{abstract}
Avoiding, Pretending, and Querying: Three Combinatorial Problems

Adam S. Jobson
\end{abstract}

18 November 2011

A $k$-term quasi-progression of diameter $d$ is a sequence $\left\{x_{1}, \ldots, x_{k}\right\}$ for which there exists a positive integer $l$ such that $l \leq x_{i}-x_{i-1} \leq l+d$, for all $i=2, \ldots, k$. Quasi-progressions may be thought of as arithmetic progressions with a certain amount of 'wiggle-room' allowed. Let $Q(d, k)$ be the least positive integer such that every 2 -coloring of $\{1, \ldots, Q(d, k)\}$ contains a monochromatic $k$-term quasi-progression of diameter $d$. We prove a conjecture of Landman for certain values of $k$ and $d$, provide counterexamples for some other cases, and determine that the conjecture always has the correct order of growth.

Let $A$ be the adjacency matrix of a nonempty graph. Is there always a nonzero $\{0,1\}$ vector in the row space of $A$ that is not a row of $A$ ? Akbari, Cameron, and Khosrovshahi have shown that an affirmative answer to this question would imply bounds on many graph parameters as a function of the rank of the adjacency matrix. We demonstrate the existence of such vectors for certain families of graphs, examine techniques to find and verify the existence of such vectors, and show that if you generalize the problem to allow asymmetry in the matrices then some $\{0,1\}$-matrices do not have such vectors.

In 1981, Andrew Yao asked "Should tables be sorted?". When the table has $n$ cells that are filled with entries taken from a key space of $m$ possibilities, he showed that it is possible to decide whether any member of the key space is present in the table by inspecting (querying) only one cell of the table if and only if $m \leq 2 n-2$. We make steps toward extending his result to the case where you are permitted two queries by considering several variations of the problem. 


\section{TABLE OF CONTENTS}

ACKNOWLEDGEMENTS $\ldots \ldots \ldots \ldots \ldots \ldots \ldots \ldots$ iv

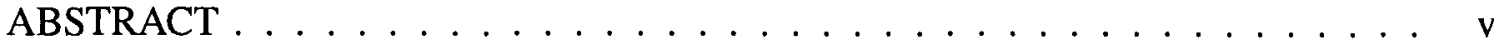

1 AVOIDING: COLORING QUASI-PROGRESSIONS $\ldots \ldots \ldots \ldots$

1.1 Introduction $\ldots \ldots \ldots \ldots \ldots \ldots \ldots \ldots \ldots$

1.2 Superblocks $\ldots \ldots \ldots \ldots \ldots \ldots \ldots \ldots \ldots \ldots \ldots \ldots$

1.3 Some upper bounds $\ldots \ldots \ldots \ldots \ldots \ldots$

1.4 A general lower bound $\ldots \ldots \ldots \ldots \ldots$

1.5 Computational Results . . . . . . . . . . . . . . . . 16

2 PRETENDING: THE ROW SPACE OF AN ADJACENCY MATRIX . . . . . 20

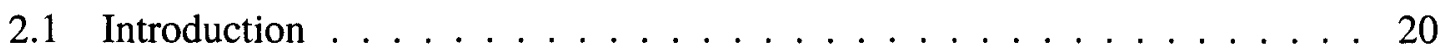

2.2 Basic Results . . . . . . . . . . . . . . . . . . . . . 22

2.3 Generalization . . . . . . . . . . . . . . . . . . . 27

2.4 Searching and Verification $\ldots \ldots \ldots \ldots \ldots$

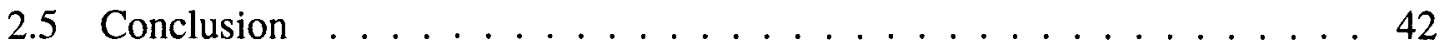

3 QUERYING: YAO'S PROBLEM . . . . . . . . . . . . . . . . . . . . 43

3.1 Introduction $\ldots \ldots \ldots \ldots \ldots \ldots \ldots \ldots \ldots \ldots \ldots \ldots \ldots$

3.2 Two queries . . . . . . . . . . . . . . . . . . 47

3.3 Graph Queryability . . . . . . . . . . . . . . . 51

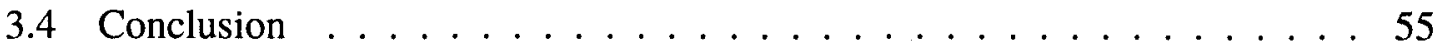

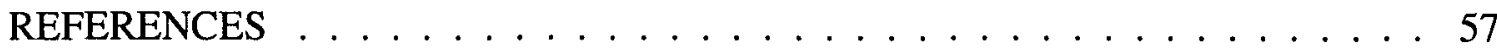

GLOSSARY OF NOTATION . . . . . . . . . . . . . . . . . . . . . . . 59

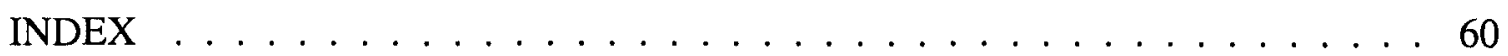

CURRICULUM VITE $\ldots \ldots \ldots \ldots \ldots$. . . . . . . . . . . . . 62 


\section{CHAPTER 1}

\section{AVOIDING: COLORING QUASI-PROGRESSIONS}

Several ${ }^{1}$ renowned open conjectures in combinatorics and number theory involve arithmetic progressions. Van der Waerden famously proved in 1927 that for each positive integer $k$ there exists a least positive integer $w(k)$ such that any 2-coloring of $1, \ldots, w(k)$ produces a monochromatic $k$-term arithmetic progression. The best known upper bound for $w(k)$ is due to Gowers and is quite large. Ron Graham [6] conjectures $w(k) \leq 2^{k^{2}}$, for all $k$.

\section{1 - Introduction}

Brown, Erdős, and Freedman [3] introduced quasi-progressions as a way of generalizing arithmetic progressions:

Definition 1.1 (Quasi-progression). A $k$-term quasi-progression (QP) of diameter $d$ is a sequence $\left\{x_{1}, \ldots, x_{k}\right\}$ for which there exists a positive integer $l$ such that $l \leq x_{i}-x_{i-1} \leq l+d$, for all $i=2, \ldots, k$.

$l$ is called the low-difference of the QP.

Arithmetic progressions are quasi-progressions with diameter zero. Analogous to the van der Waerden function $w(k)$, Landman $[8,9]$ introduced a Ramsey function for quasiprogressions.

Definition 1.2. $Q(d, k)$ is the least positive integer such that every two-coloring of the set $\{1, \ldots, Q(d, k)\}$ contains a monochromatic $k$-term quasi-progression of diameter $d$.

This function produces a lower bound for $w(k)$ since

$$
w(k)=Q(0, k) \geq Q(1, k) \geq Q(2, k) \geq Q(3, k) \geq \cdots
$$

\footnotetext{
${ }^{1}$ This chapter contains joint work with André Kézdy, Hunter Snevily, and Susan White, and has been submitted to a journal for publication.
} 
So, it is of great interest to find bounds on $Q(d, k)$ for various $d$, especially small values of $d$. Of particular interest is the rate of growth of $Q(1, k)$. Is it merely polynomial or is it at least exponential in $k$ ? Vijay [13] $]^{2}$ has recently established an exponential lower bound for $Q(1, k)$, so quasi-progressions of small diameter behave similarly to arithmetic progressions, at least with respect to these Ramsey functions. An interesting open problem is to determine the largest diameter $d$ for which $Q(d, k)$ is at least exponential (in $k$ ).

Landman established several bounds on $Q(d, k)$ and made several conjectures which we resolve in this paper. Our results, like Landman's, focus on large diameter; that is, $d=k-i$, for some positive integer $i$ satisfying $k \geq 2 i \geq 1$. The main difficulty is the upper bound on $Q(k-i, k)$. Specifically, how can we tailor an argument that handles the large number of extremal 2-colorings which seem to defy uniform description (cf. Landman's data at the end of his paper)? In section 1.2 we introduce superblocks, an equivalence relation that imposes sufficient structure on extremal 2-colorings to extract long monochromatic quasi-progression fragments via a greedy strategy. Through the superblock lens, the extremal 2-colorings coalesce. The superblock argument is used in section 1.3 where we show how to splice monochromatic fragments together to produce long monochromatic quasiprogressions. This yields an upper bound on $Q(k-i, k)$; the bound is often sharp. One consequence is that, if $k \geq 2 i$ and $k=m i+r$ for integers $m, r$ such that $1<r<i$, then

$$
Q(k-i, k) \leq 2 i k-4 i+2 r-1 \text {. }
$$

This improves bounds given by Landman.

Our main result is proven is section 1.4:

$$
Q(k-i, k)=2 i k-4 i+2 r-1,
$$

if $k=m i+r$ for integers $m, r$ such that $3 \leq r<\frac{i}{2}$ and $r-1 \leq m$. This disproves Conjecture 1 of Landman's paper for these values of $r$. Residues $r \geq i / 2$ that are not considered in this

\footnotetext{
${ }^{2}$ We thank Bruce Landman for bringing our attention to Vijay's paper.
} 
result appear to be more difficult. The techniques here are inadequate to resolve those values of $Q(k-i, k)$. However, we also prove that, if $k \geq 2 i \geq 1$, then

$$
Q(k-i, k)= \begin{cases}2 i k-4 i+3 & k \equiv 0 \text { or } 2 \quad(\bmod i) \\ 2 i k-2 i+1 \quad k \equiv 1 \quad(\bmod i)\end{cases}
$$

thus proving Landman's conjecture in these cases.

\section{2 - Superblocks}

In this section we develop notation, concepts and tools to describe the structure of extremal strings which avoid long monochromatic quasi-progressions.

A $k$-term progression is an increasing sequence of $k$ positive integers $x_{1}<x_{2}<\cdots<x_{k}$. Given a $k$-term progression $P=\left\{x_{j}\right\}_{j=1}^{k}$, the differences in $P$ are the elements in the set

$$
D(P)=\left\{x_{j}-x_{j-1}: j=2, \ldots, k\right\}
$$

The low-difference of $P$, denoted $\delta(P)$ (or simply $\delta$ ), is the minimum element in $D(P)$; the high-difference of $P$, denoted $\Delta(P)$ (or simply $\Delta$ ), is the maximum. The diameter of $P$ is $d=\Delta-\delta$. Observe that arithmetic progressions are quasi-progressions with diameter $d=0$.

Consider now 2-colorings of the positive integers. A quasi-progression is a good progression if it is a monochromatic quasi-progression with length $k$ and diameter at most $d$. Define $Q(d, k)$ to be the least positive integer such that every 2-coloring of $\{1, \ldots, Q(d, k)\}$ contains a good progression. Motivated by conjectures of Landman [8], we consider $Q(d, k)$ for values of $d$ and $k$ satisfying $d=k-i$ and $k \geq 2 i$, where $i$ is some fixed positive integer. So, for the rest of this paper $d=k-i$ and a good progression means a monochromatic quasi-progression with length $k$ and diameter at most $k-i$. To understand the structure of extremal 2-colorings avoiding good progressions, we now introduce two important substructures: blocks and superblocks. 
Let $C=c_{1} c_{2} \ldots c_{\ell}$ be a binary string of length $\ell$. A substring of $C$ is a string of the form $c_{p} c_{p+1} \cdots c_{q}$, for some positive integers $1 \leq p \leq q \leq \ell$. A block of $C$ is a maximal monochromatic substring of $C$. We employ the usual shorthand notation in which, for $x \in$ $\{0,1\}$, the shorthand $x^{n}$ represents the string $\underbrace{x \cdots x}_{n}$. There is a natural partition of $C$ into blocks: without loss of generality, the first block of $C$ is a block of $1 \mathrm{~s}$ so

$$
C=1^{\alpha_{1}} 0^{\alpha_{2}} \cdots 1^{\alpha_{b-1}} 0^{\alpha_{b}}
$$

where the $\alpha_{j}$ are positive integers, except possibly $\alpha_{b}$ which may be zero (in which case the final block of $C$ is actually a block of 1 s). Note that $\sum_{j=1}^{b} \alpha_{j}=\ell$.

Now consider an extremal coloring $C$; that is, suppose that $\ell=Q(k-i, k)-1$ and $C$ represents a 2 -coloring of the integers $1, \ldots, \ell$ with no good progression. For convenience, blocks of $C$ of length at most $k-i$ are minor blocks; longer blocks are major. There are two important facts that motivate this dichotomy:

(i) Quasi-progressions with low-difference 1 and diameter $k-i$ can jump over any intermediate minor blocks.

(ii) "Greedy monochromatic jumping" (in which jumps of length at least $\delta$ but at most $\delta+k-i$ are taken, for some choice of $\delta$ ) can not get stuck in substrings that avoid major blocks of one color (see later Theorem 1.6 and Theorem 1.7).

A consequence of observation (i) is that, in a substring in which only minor blocks of one color appear, all of the integers with the other color in this substring form a monochromatic progression with low-difference 1 and high-difference $k-i+1$ (that is, a monochromatic quasi-progression with diameter $k-i$ ).

Now we turn to the task of identifying monochromatic substructures of $C$ with the property that a greedy strategy can guarantee dense monochromatic progressions beginning and ending at endpoints of the substructure. To make this precise, we define the equivalence relation $\sim$ on the integers $1, \ldots, \ell$ so that $x \sim y$ if and only if $x$ and $y$ are contained in a monochromatic quasi-progression $P$ of $C$ such that $D(P) \subseteq\{1, \ldots, k-i+1\}$ (the transitivity of $\sim$ follows from the fact that the union of two intersecting monochromatic quasi- 
progressions with differences in $\{1, \ldots, k-i+1\}$ is another such quasi-progression). The equivalence classes under $\sim$ are called superblocks. Superblocks are not necessarily substrings. Suppose that $C$ has $t$ superblocks $B_{1}, \ldots, B_{t}$. We naturally order superblocks this way: $B_{p}<B_{q}$ if and only if $\min B_{p}<\min B_{q}$, where $\min B_{p}$ denotes the smallest integer in $B_{p}$ (that is, the left-most one). A superblock is major if it contains all of the elements from a major block of $C$; otherwise it is minor. The extremes of a superblock are its minimum and maximum elements.

Example 1.3. Consider $k=12, i=6$. Because $Q(6,12)=123$, an extremal string in this case has length 122 . There are several extremal strings, one is shown below:

$$
C=1^{6} 0^{10} 1^{1} 0^{1} 1^{10} 0^{11} 1^{11} 0^{10} 1^{1} 0^{1} 1^{10} 0^{10} 1^{1} 0^{1} 1^{10} 0^{11} 1^{11} 0^{6}
$$

This string has 18 blocks, but only 12 superblocks of which exactly two are minor. The cardinalities of the superblocks are (in this order)

$$
6,11,11,11,11,11,11,11,11,11,11,6
$$

Theorem 1.4 (Superblock Upper Bound). If $C$ is a 2-coloring with no good progression, then every superblock of $C$ has cardinality at most $k-1$.

Proof. The union of two intersecting monochromatic quasi-progression of $C$ using differences from $\{1, \ldots, k-i+1\}$ is also a monochromatic quasi-progression of $C$ using differences from $\{1, \ldots, k-i+1\}$. It follows that all of the elements of a superblock are contained in a single monochromatic quasi-progression of $C$ using differences from $\{1, \ldots, k-i+1\}$. Because $C$ contains no good quasi-progression, each superblock contains fewer than $k$ elements.

The following theorem lists basic facts about superblocks.

Theorem 1.5 (Superblock Fundamentals). Suppose $C$ is a binary string that represents a 2-coloring with no good progression. If $C$ has $t$ superblocks $B_{1}<\cdots<B_{t}$, then

(i) all elements in a superblock have the same color (that is, superblocks are monochromatic), 
(ii) $B_{1}, \ldots, B_{t}$ form a partition of $C$,

(iii) consecutive superblocks have opposite color,

(iv) superblocks $B_{2}, \ldots, B_{t-1}$ contain exactly one major block (in particular they are major superblocks),

(v) an extreme element of a superblock is adjacent to either the end of the string, a minor superblock, or the major block of neighboring major superblock, and

(vi) a substring of $C$ consisting of all characters between (and including) the extreme elements of a superblock contains exactly one major block.

Proof. (i) two elements are in relation $\sim$ if they are in a common monochromatic quasiprogression. Therefore their color is identical. (ii) the equivalence classes of an equivalence relation form a partition. (iii)-(vi) the boundary of a superblock is reached at the end of the string or at an obstructing major block of the opposite color. Thus each superblock with a neighbor, must contain a major block that defines the boundary of that neighbor. So superblocks alternate color. If a superblock contained two major blocks, then its size would exceed $k-1$, contradicting Theorem 1.4.

Note that, by (ii), the length of $C$ is $\ell=\sum_{j=1}^{t}\left|B_{j}\right|$. The basic approach for an upper bound on the length of $C$ is based on this partition - we seek to bound the cardinality of each of the superblocks. To accomplish this, we need to argue that segments of a long monochromatic quasi-progression can be strung together using fragments from each superblock. The technique relies on the following two fundamental theorems.

Theorem 1.6 (Greedy Major Superblock Jumping). Assume $k \geq 2 i$. If $B$ is a major superblock of $C$ and $1 \leq \delta \leq i$, then $B$ contains a monochromatic quasi-progression $P$ such that

(i) $P$ has length at least $\left\lceil\frac{|B|}{\delta}\right\rceil$,

(ii) the low difference of $P$ is at least $\delta$,

(iii) the diameter of $P$ is at most $k-i$, and

(iv) both extremes of $B$ are in $P$. 
Proof. Without loss of generality, $B$ has color 1 . Let $S$ denote the binary substring of $C$ consisting of all characters between the minimum and maximum elements of $B$. By definition, $S$ begins and ends with a 1 . Let us suppose that $p \delta<|B| \leq(p+1) \delta$, for some positive integer $p$. We must show that there is a monochromatic quasi-progression of length at least $p+1$ (that is, a progression that satisfies (i) above) with the additional properties (ii)-(iv). First, create a monochromatic quasi-progression this way: start with the left-most 1 of $S$ and repeatedly jump right to the first available 1 that is distance at least $\delta$, but no more than $\delta+k-i$ from the last chosen 1 . Note that there can never be an obstruction to jumping to the next available 1 unless we reach the end of $S$ because, if a jump to the next 1 required a length more than $\delta+k-i$, then the last $k-i+1$ skipped elements would be a major block of $0 \mathrm{~s}$ in $S$ which is impossible by Theorem 1.5 (vi). This means that when this greedy jumping reaches the end of $S$, it must land on a 1 that is distance at most $\delta-1$ of the rightmost 1 of $S$. Also notice that each jump can pass over at most $\delta-1$ ones. Thus each 1 in our constructed progression "consumes" at most $\delta$ ones: itself plus the at most $\delta-1$ ones that are skipped by the next jump. But, since there are more than $p \delta$ ones and we have not wasted any $1 \mathrm{~s}$ because we started at the beginning of $S$, our progression must have at least $p+1$ ones. The only problem is that this progression may not end at the maximum element of $B$. We now address this problem.

Let $x$ denote the leftmost 1 of $S$ and $y$ the rightmost 1 of $S$. Suppose that our currently constructed progression from $S$ is $x=x_{1}<\cdots<x_{q}$, for some $q \geq p+1$. In a manner similar to the construction of this sequence, construct a new progression starting at $y$ and greedily jumping leftward toward $x$. Suppose that this second progression is $y_{h}<\cdots<y_{2}<y_{1}$; that is, this progression begins at the rightmost element $y_{1}=y$ of $S$, jumps leftwards greedily until it reaches $y_{h}$ and no further jumps are possible. A consequence of the next claim is that the $x$-progression and the $y$-progression have the same length (i.e. $h=q$ ).

Claim: $y_{j}-x_{q+1-j}<\delta$, for $j=1, \ldots, q$.

We prove this by induction on $j$. The basis case is true because $y_{1}=y$ and as noted 
in the paragraph above, the progression of $x$ s must end within $\delta$ of $y$. Now suppose that $y_{j}-x_{q+1-j}<\delta$, for some $j$. Because the progression of $y$ s must end within $\delta$ of $x$, if $q+1-j>$ 1 , the element $y_{j+1}$ must exist. The distance $y_{j}-y_{j+1}$ must be at least $\delta$ so $y_{j+1}<x_{q+1-j}$. In particular, $x_{q-j} \leq y_{j+1}<x_{q+1-j}$. Because the $x$-sequence did not jump from $x_{q-j}$ to $y_{j+1}$, it follows that $y_{j+1}-x_{q-j} \leq \delta-1$, as desired.

Observe that if $y_{j}=x_{q+1-j}$, for some $j \in\{1, \ldots, q\}$ then the progression

$$
x_{1}, \ldots, x_{q+1-j}, y_{j-1}, \ldots, y_{1}
$$

satisfies the conclusion of the theorem.

So, we have proven that we may assume that these two sequences interlace:

$$
x_{1}<y_{q}<x_{2}<y_{q-1}<\cdots<x_{q-1}<y_{2}<x_{q}<y_{1}
$$

and $y_{j}-x_{q+1-j}<\delta$, for $j=1, \ldots, q$.

Now let $T$ denote the substring of $S$ corresponding to the major block of $1 \mathrm{~s}$ in $B$. Because $T$ is a major block, $T$ is a substring of $1 \mathrm{~s}$ with length at least $k-i+1$. In particular, since $k \geq 2 i$, the length of $T$ is at least $i+1$, which is larger than $\delta$. Now observe that among the differences between consecutive elements of the progression $x_{1}, \ldots, x_{q}$, there must be a difference of exactly $\delta$ because the first greedy jump that this progression makes into $T$ must either have length exactly $\delta$ or it hits the first element of $T$. In the latter event, the following jump must have length $\delta$ because $T$ contains at least $\delta+1$ ones.

So, there is some $j \in\{1, \ldots, q-1\}$ such that $x_{q+1-j}-x_{q-j}=\delta$. Because $y_{j}-x_{q+1-j}<\delta$, it follows that $y_{j}-x_{q-j} \leq 2 \delta \leq k-i+\delta$. Therefore, the progression

$$
x_{1}, \ldots, x_{q-j}, y_{j}, \ldots, y_{1}
$$

satisfies the conclusion of the theorem.

Theorem 1.7 (Greedy Minor Superblock Jumping). Assume $k \geq 2 i$. If $B$ is a minor superblock of $C, x$ is an extreme of $B$, and $1 \leq \delta \leq i$, then $B$ contains a monochromatic quasi-progression $P$ such that 
(i) $P$ has length at least $\left\lceil\frac{|B|}{\delta}\right\rceil$,

(ii) the low difference of $P$ is at least $\delta$,

(iii) the diameter of $P$ is at most $k-i$, and

(iv) $x \in P$.

Proof. We argue essentially the same way as in the proof of Theorem 1.6. Without loss of generality, $B$ has color 1 and $x$ is the leftmost element of $B$ (that is, $x=\min B$ ). Let $S$ denote the binary substring of $C$ consisting of all characters between the minimum and maximum elements of $B$. By definition, $S$ begins and ends with a 1 . Let us suppose that $p \delta<|B| \leq(p+1) \delta$, for some positive integer $p$. We must show that there is monochromatic quasi-progression of length at least $p+1$ (that is, a progression that satisfies (i) above) with the additional properties (ii)-(iv). Create such a monochromatic quasi-progression this way: start with $x$ and repeatedly jump right to the first available 1 that is distance at least $\delta$, but no more than $\delta+k-i$ from the last chosen 1 . Note that there can never be an obstruction to jumping to the next available 1 unless we reach the end of $S$ because, if a jump to the next 1 required a length more than $\delta+k-i$, then the last $k-i+1$ skipped elements would be a major block of $0 \mathrm{~s}$ in $S$ which is impossible by Theorem 1.5 (vi). This means that when this greedy jumping reaches the end of $S$, it must lands on a 1 that is distance at most $\delta-1$ of the right most 1 of $S$. Also notice that each jump can pass over at most $\delta-1$ ones. Thus each 1 in our constructed progression "consumes" at most $\delta$ ones, itself plus the at most $\delta-1$ ones that are skipped by the next jump. But, since there are more than $p \delta$ ones and we have not wasted any $1 \mathrm{~s}$ because we started at the beginning of $S$, our progression must have at least $p+1$ ones.

The next theorem brings together the previous two theorems and is a significant tool in later proofs.

Theorem 1.8. Assume $k \geq 2 i$. Suppose $C$ is a binary string that represents a $\{$ red, blue $\}$ coloring of positive integers with no good progression. If $C$ has no red major blocks of cardinality at least $k-i+\delta$, for some $1 \leq \delta \leq i$, and $B_{1}, \ldots, B_{h}$ are the blue superblocks 
of $C$, then $C$ contains a monochromatic quasi-progression $P$ with diameter at most $k-i$, low-difference at least $\delta$, and length at least $\sum_{j=1}^{h}\left\lceil\frac{\left|B_{j}\right|}{\delta}\right\rceil$.

Proof. Apply Theorem 1.6 to superblocks $B_{2}, \ldots, B_{h-1}$ to obtain monochromatic quasiprogression fragments $P_{2}, \ldots, P_{h-1}$ with length at least $\left\lceil\frac{\left|B_{j}\right|}{\delta}\right\rceil$, low-difference $\delta$, diameter $k-i$ and that contain both extremes of each of their major superblocks. Similarly, apply Theorem 1.7 to superblocks $B_{1}$ and $B_{h}$ to obtain monochromatic quasi-progression fragments $P_{1}$ and $P_{h}$ with length at least $\left\lceil\frac{\left|B_{j}\right|}{\delta}\right\rceil$, low-difference $\delta$, diameter $k-i$ and that contain the maximum and minimum, respectively, of $B_{1}$ and $B_{h}$. Because $C$ has no red major blocks of cardinality at least $k-i+\delta$, jumps of length at most $k-i+\delta$ (and at least $\delta$ ) can be made to join the extremes of these fragments into the desired monochromatic quasi-progression $P$.

\section{3 - Some upper bounds}

This section establishes upper bounds on $Q(k-i, k)$. In many cases the bounds are sharp. The proofs rely heavily on the superblock results from the previous section.

Theorem 1.9. If $k \geq 2 i$ and $k \equiv 0(\bmod i)$, then $Q(k-i, k)=2 i k-4 i+3$. Proof. The lower bound $Q(k-i, k) \geq 2 i k-4 i+3$ follows from Corollary 1 of Landman's paper [8]; so it suffices to prove the upper bound. Suppose that $\ell=Q(k-i, k)-1$ and $C$ is a binary string of length $\ell$ that represents a 2 -coloring of the integers $1, \ldots, \ell$ with no good progression. We must prove that $\ell \leq 2 i k-4 i+2$. Assume that $k=m i$, for some $m \geq 2$.

We claim that there can not be $i$ major superblocks of the same color. To see this, suppose to the contrary, that $B_{1}, \ldots, B_{p}(p \geq i)$ are all of the blue major superblocks of $C$. Apply Theorem 1.8 with $\delta=i$ to the substring of $C$ between the $\min B_{1}$ and the $\max B_{i}$. This theorem guarantees a monochromatic quasi-progression of length at least

$$
\sum_{j=1}^{p}\left\lceil\frac{\left|B_{j}\right|}{i}\right\rceil \geq \sum_{j=1}^{p}\left\lceil\frac{k-i+1}{i}\right\rceil \geq \sum_{j=1}^{p} m \geq m p \geq k
$$

a contradiction. So $C$ has at most $i-1$ major superblocks of each color. 
Suppose that $C$ has $i-1$ major superblocks of the same color. We now claim that the total number of elements in minor blocks of that color is at most $k-i$. To prove this, suppose that $C$ has $i-1$ blue major superblocks $B_{1}, \ldots, B_{i-1}$ and two minor superblocks $B_{0}$ and $B_{i}$ (it is clear that there are most two blue minor superblocks, since there is at most one at each end). Again, apply Theorem 1.8 with $\delta=i$ to the substring of $C$ between the $\min B_{0}$ and the $\max B_{i}$. This theorem guarantees a monochromatic quasi-progression of length at least

$$
\sum_{j=0}^{i}\left\lceil\frac{\left|B_{j}\right|}{i}\right\rceil=(i-1) m+\left\lceil\frac{\left|B_{0}\right|}{i}\right\rceil+\left\lceil\frac{\left|B_{i}\right|}{i}\right\rceil .
$$

Since this sum is at most $k-1=m i-1$, it follows that $\left|B_{0}\right|+\left|B_{i}\right| \leq k-i$. Consequently, the blue superblocks have cardinalities that sum to at most $(i-1)(k-1)+(k-i)$. The same argument applies to the red superblocks. Therefore, the length of $C$ is at most

$$
2(i-1)(k-1)+2(k-i)=2 i k-4 i+2,
$$

as desired.

Theorem 1.10. If $k \geq 2 i+1$ and $k \equiv 1(\bmod i)$, then $Q(k-i, k)=2 i k-2 i+1$.

Proof. The lower bound $Q(k-i, k) \geq 2 i k-2 i+1$ follows from Corollary 1 of Landman's paper [8]; so it suffices to prove the upper bound. Let $C$ be binary string realizing an extremal 2-coloring with no good progression. We must prove that the length of $C$ is at most $2 i(k-1)$. Let $B_{1}, \ldots, B_{p}$ be the blue superblocks of $C$ and $R_{1}, \ldots, R_{q}$ the red superblocks. Because the superblocks form a partition, the length of $C$ is $\sum_{j=1}^{p}\left|B_{j}\right|+\sum_{j=1}^{q}\left|R_{j}\right|$. However, applying Theorem 1.8 with $\delta=i$ to the substring of $C$ between the $\min B_{1}$ and the $\max B_{p}$, we find that $C$ contains a monochromatic quasi-progression of length $\sum_{j=0}^{p}\left\lceil\frac{\left|B_{j}\right|}{i}\right]$. Since this can not exceed $k-1$, it follows that $\sum_{j=1}^{p}\left|B_{j}\right| \leq i(k-1)$. A symmetric argument shows $\sum_{j=1}^{q}\left|R_{j}\right| \leq i(k-1)$. Therefore the length of $C$ is at most $2 i(k-1)$, as desired.

The next theorem gives a general upper bound that we shall show in the next section is sharp when $k=m i+r$ for integers $m, r$ such that $3 \leq r<\frac{i}{2}$ and $r-1 \leq m$.

Theorem 1.11. If $k \geq 2 i$ and $k=m i+r$ for integers $m, r$ such that $1<r<i$, then

$$
Q(k-i, k) \leq 2 i k-4 i+2 r-1 \text {. }
$$


Proof. Suppose that $\ell=Q(k-i, k)-1$ and $C$ is a binary string of length $\ell$ that represents a 2 -coloring of the integers $1, \ldots, \ell$ with no good progression. We must prove that $\ell \leq$ $2 i k-4 i+2 r-2$. We argue by contradiction: assume that $\ell>2 i k-4 i+2 r-2$.

For $j=0,1$, let $\alpha_{j}$ denoted the number of superblocks of $C$ that have size greater than $m i$. Apply Theorem 1.8 with $\delta=i$ to the shortest substring containing all superblocks of color $j$ :

$$
\alpha_{j}(m+1)+\sum_{\substack{B \text { has color } j \\|B| \leq m i}}\left\lceil\frac{|B|}{i}\right\rceil \leq k-1 .
$$

It follows that, for $j=0,1$,

$$
\sum_{\substack{B \text { has color } j \\|B| \leq m i}}|B| \leq i\left(k-1-\alpha_{j}(m+1)\right) .
$$

Therefore, the length of $C$ can be bounded as follows:

$$
\begin{aligned}
\ell & =\sum_{j=0}^{1}\left(\sum_{\substack{B \text { has color } j \\
|B|>m i}}|B|\right)+\sum_{j=0}^{1}\left(\sum_{\substack{B \text { has color } j \\
|B| \leq m i}}|B|\right) \\
& \leq\left(\alpha_{0}+\alpha_{1}\right)(k-1)+i\left(k-1-\alpha_{0}(m+1)\right)+i\left(k-1-\alpha_{1}(m+1)\right) \\
& =2 i k-2 i-\alpha(i+1-r),
\end{aligned}
$$

where $\alpha=\alpha_{0}+\alpha_{1}$. Because we are assuming that $\ell>2 i k-4 i+2 r-2$, we may conclude that $\alpha<2$. Without loss of generality, $\alpha_{0}=0$ and $\alpha_{1} \leq 1$.

Because $\alpha_{0}=0$, the coloring contains no superblocks of 0 s with cardinality larger than $m i$. Now apply Theorem 1.8 with $\delta=i-r+1$ to the substring of $C$ containing all superblocks of color 1:

$$
\sum_{B \text { has color } 1}\left\lceil\frac{|B|}{i-r+1}\right\rceil \leq k-1 .
$$

Consequently the number of $1 \mathrm{~s}$ in $C$ is at most $(k-1)(i-r+1)$. Applying Theorem 1.8 with $\delta=i$ to the substring of $C$ containing all superblocks of color 0 we find

$$
\sum_{B \text { has color } 0}\left\lceil\frac{|B|}{i}\right\rceil \leq k-1
$$


Therefore the number of $0 \mathrm{~s}$ in $C$ is at most $(k-1) i$. It follows that the length of $C$ can be bounded as follows:

$$
\begin{aligned}
\ell & =(\# 1 \mathrm{~s} \text { in } C)+(\# 0 \mathrm{~s} \text { in } C) \\
& \leq(k-1)(i-r+1)+(k-1) i \\
& =2 i k-2 i+(k-1)(1-r),
\end{aligned}
$$

which is at most $2 i k-4 i+2 r-2$ since $r>1$ and $k>2 i$. This contradicts that $\ell>$ $2 i k-4 i+2 r-2$

\section{4 - A general lower bound}

In this section we exhibit an extremal 2-coloring of positive integers avoiding monochromatic $k$-term quasi-progressions of diameter $k-i$ for many values of $k$ and $i$. To describe this coloring we first introduce some notation.

Recall that a block of a binary string is a maximal length monochromatic substring. A segment of a binary string is a maximal length substring in which all blocks have the same length. Its segments naturally partition a binary string. Therefore a binary string $C$ can be abbreviated by an expression involving positive integers of the form $a_{1}^{b_{1}} \cdots a_{s}^{b_{s}}$, which indicates that the $j$ th segment consists of $b_{j}$ blocks of length $a_{j}$ (we assume that the first block is a block of 1s). We adopt this notation in this section. Note that $C$ has length $\sum_{j=1}^{s} a_{j} b_{j}$.

Theorem 1.12. Suppose that $k=m i+r$ for integers $m, r$ such that $3 \leq r<\frac{i}{2}$. If $r-1 \leq$ $m$, then the following 2 -coloring of the integers from 1 to $2 i k-4 i+2 r-2$ contains no monochromatic $k$-term quasi-progression of diameter $k-i$ :

$$
(i(r-2))^{1}(m i)^{i-1}(k-1)^{2}(m i)^{i-1}(i(r-2))^{1} .
$$

Proof. Let $C$ denote the binary string corresponding to this coloring. We assume that $C$ begins with a block of $1 \mathrm{~s}$. Observe that $C$ is a palindrome and, because $r-1 \leq m$, the initial block of $1 \mathrm{~s}$ is shorter than the others. For a positive integer $\delta$, let $Q_{\delta}$ be a longest monochromatic quasi-progression in $C$ with low-difference $\delta$, and let $\ell$ be the length of $Q_{\delta}$. 
We must show $\ell \leq k-1$. Because $C$ is a palindrome, we may assume that $Q_{\delta}$ consists of elements of color 1 . If $\delta<i-r+1$, then $Q_{\delta}$ can not jump across blocks of length $m i$ or longer, so $Q_{\delta}$ has length at most $k-1$ in this case. So it suffices to consider values of $\delta \geq i-r+1$. However, because $C$ has only interior blocks of length $m i$ or $k-1$, we can restrict our attention of $\delta=i-r+1$ (which permits leaps over blocks size $m i$ but not $k-1$ ) or $\delta=i$ (which permits leaps over all block sizes). Upper bounds on $\ell$ for other values of $\delta$ follow immediately from the upper bounds on these two values of $\delta$.

Assume first that $\delta=i$. The quasi-progression $Q_{\delta}$ can use at most $\left[\frac{|B|}{i}\right]$ elements from a block $B$. Consequently, the length $\ell$ can be bounded this way:

$$
\begin{aligned}
\ell & \leq\left\lceil\frac{i(r-2)}{i}\right\rceil+(i-1)\left\lceil\frac{m i}{i}\right\rceil+\left\lceil\frac{k-1}{i}\right\rceil \\
& =(r-2)+(i-1) m+(m+1) \\
& =k-1
\end{aligned}
$$

as desired.

Assume now that $\delta=i-r+1$. Clearly the quasi-progression $Q_{\delta}$ can use at most $\left[\frac{|B|}{i-r+1}\right\rceil$ elements from a block $B$. There are two cases according to the parity of $i$. In each case the length of $Q_{\delta}$ is bounded from above:

If $i$ is even the extremal length occurs when $Q_{\delta}$ uses $1 \mathrm{~s}$ from the small beginning block, $(i-2) / 2$ intermediate blocks of size $m i$, and the large block of $k-1$ ones, so

$$
\ell \leq\left\lceil\frac{i(r-2)}{i-r+1}\right\rceil+\frac{i-2}{2}\left\lceil\frac{m i}{i-r+1}\right\rceil+\left\lceil\frac{k-1}{i-r+1}\right\rceil .
$$

If $i$ is odd the extremal length occurs when $Q_{\delta}$ uses $1 \mathrm{~s}$ from $(i-1) / 2$ intermediate blocks of size $m i$, and the large block of $k-1$ ones, so

$$
\ell \leq \frac{i-1}{2}\left\lceil\frac{m i}{i-r+1}\right\rceil+\left\lceil\frac{k-1}{i-r+1}\right\rceil .
$$

Consider now the following upper bounds which, when substituted into inequalities (1.1) and (1.2), will show that $\ell \leq k-1$. 
Bound 1: $\left\lceil\frac{m i}{i-r+1}\right\rceil \leq \min \left\{2 m-1,2 m+\frac{2(r-1-m)}{i-1}\right\}$.

It suffices to prove that $\frac{m i}{i-r+1} \leq 2 m-1+\frac{2(r-1-m)}{i-1}$. Assume the contrary: $\frac{m i}{i-r+1}>$ $2 m-1+\frac{2(r-1-m)}{i-1}$. Applying the assumption that $2 r+1 \leq i$ while solving for $m$, we find that

$$
\frac{(i-r+1)(i-2 r+1)}{i(i-2 r-1)+4(r-1)}>m
$$

Because we have assumed that $m \geq r-1$, it follows that

$$
\frac{(i-r+1)(i-2 r+1)}{i(i-2 r-1)+4(r-1)}>r-1 \text {. }
$$

Consequently,

$$
\begin{aligned}
0 & >(r-1)[i(i-2 r-1)+4(r-1)]-(i-r+1)(i-2 r+1) \\
& =(r-2)(i-1)\left(i-\frac{2 r^{2}-5 r+3}{r-2}\right)
\end{aligned}
$$

Because $r>2$ and $i>1$, we conclude that $i<\frac{2 r^{2}-5 r+3}{r-2}$. However, since $i>2 r$ we find that

$$
2 r<\frac{2 r^{2}-5 r+3}{r-2}
$$

which implies that $r<3$, a contradiction.

Bound 2: $\left\lceil\frac{k-1}{i-r+1}\right\rceil \leq 2 m$.

Assume, to the contrary, that $\frac{k-1}{i-r+1}>2 m$. Substituting $k=m i+r$ and solving for $m$ produces the inequality $\frac{r-1}{i-2 r+2}>m$. Because we have assumed that $m \geq r-1$, it follows that $\frac{r-1}{i-2 r+2}>r-1$ which implies that $i<2 r-1$, a contradiction.

Bound 3: $\left\lceil\frac{i(r-2)}{i-r+1}\right\rceil \leq \frac{i}{2}+r-2$.

It suffices to prove that $\frac{i(r-2)}{i-r+1} \leq \frac{i}{2}+r-3$. Assume, to the contrary, that $\frac{i(r-2)}{i-r+1}>\frac{i}{2}+r-3$. Equivalently,

$$
0>2\left(\frac{i}{2}+r-3\right)(i-r+1)-2 i(r-2)=(i+r-3)(i-2 r+2),
$$

a contradiction. 
To complete the proof we show, using these bounds applied to inequalities (1.1) and (1.2), that $\ell \leq k-1$. Consider inequality (1.1):

$$
\begin{aligned}
\ell & \leq\left\lceil\frac{i(r-2)}{i-r+1}\right\rceil+\frac{i-2}{2}\left\lceil\frac{m i}{i-r+1}\right\rceil+\left\lceil\frac{k-1}{i-r+1}\right\rceil \\
& \leq\left(\frac{i}{2}+r-2\right)+\left(\left(\frac{i-2}{2}\right)(2 m-1)\right)+(2 m) \\
& =k-1
\end{aligned}
$$

Now consider inequality (1.2):

$$
\begin{aligned}
\ell & \leq \frac{i-1}{2}\left\lceil\frac{m i}{i-r+1}\right\rceil+\left\lceil\frac{k-1}{i-r+1}\right\rceil \\
& \leq\left(\left(\frac{i-1}{2}\right)\left(2 m+\frac{2(r-1-m)}{i-1}\right)\right)+(2 m) \\
& =k-1
\end{aligned}
$$

Theorem 1.13. If $k=m i+r$ for integers $m, r$ such that $3 \leq r<\frac{i}{2}$ and $r-1 \leq m$, then

$$
Q(k-i, k)=2 i k-4 i+2 r-1 \text {. }
$$

Proof. Theorem 1.11 proves the upper bound and Theorem 1.12 proves the lower bound.

Theorem 1.14. If $k \geq 2 i$ and $k \equiv 2(\bmod i)$, then $Q(k-i, k)=2 i k-4 i+3$.

Proof. Theorem 1.11 gives the upper bound $Q(k-i, k) \leq 2 i k-4 i+3$. Arguing in a manner similar to the proof of Theorem 1.12, one can show that

$$
(k-2)^{i-1}(k-1)^{2}(k-2)^{i-1} .
$$

is a 2 -coloring of $1, \ldots, 2 i k-4 i+2$ that avoids monochromatic $k$-term quasi-progressions of diameter $k-i$.

\section{5 - Computational Results}

Landman [8] gave a table of maximal known 2-colorings avoiding quasi-progressions of length $k$ and diameter $k-i$ for various values of $k$ and $i$. Here we present extended versions 
Table 1.1: Verified and updated version of the table from [8] $(i=3)$

\begin{tabular}{rrrl}
\hline$k$ & $i$ & $Q(k-i, k)$ & \multicolumn{1}{c}{ Maximal Valid Colorings } \\
\hline 3 & 3 & 9 & $2^{4}, 1^{3} 2^{1} 1^{3}, 1^{1} 2^{3} 1^{1}$ \\
4 & 3 & 19 & $3^{6}$ \\
5 & 3 & 29 & $4^{2} 3^{2} 4^{2} 3^{2}, 3^{2} 4^{2} 3^{2} 4^{2}, 4^{1} 3^{2} 4^{2} 3^{2} 4^{1}, 3^{1} 4^{2} 3^{2} 4^{2} 3^{1}$ \\
\hline 6 & 3 & 27 & $3^{1} 5^{4} 3^{1}$ \\
7 & 3 & 37 & $6^{6}, 3^{1} 6^{5} 3^{1}$ \\
8 & 3 & 39 & $6^{2} 7^{2} 6^{2}$ and 5 others \\
9 & 3 & 45 & $6^{1} 8^{4} 6^{1}$ \\
10 & 3 & 55 & $9^{6}, 6^{1} 9^{5} 3^{1}, 3^{1} 9^{5} 6^{1}$ \\
11 & 3 & 57 & $9^{2} 10^{2} 9^{2}$ and 7 others \\
12 & 3 & 63 & $9^{1} 11^{4} 9^{1}$ \\
13 & 3 & 73 & $12^{6}, 9^{1} 12^{5} 3^{1}, 6^{1} 12^{5} 6^{1}, 3^{1} 12^{5} 9^{1}$ \\
14 & 3 & 75 & $12^{2} 13^{2} 12^{2}$ and 9 others \\
15 & 3 & 81 & $12^{1} 14^{4} 12^{1}$ \\
16 & 3 & 91 & $15^{6}, 12^{1} 15^{5} 3^{1}, 9^{1} 15^{5} 6^{1}, 6^{1} 15^{5} 6^{1}, 3^{1} 15^{5} 12^{1}$ \\
17 & 3 & 93 & $15^{2} 16^{2} 15^{2}$ and 11 others \\
$\mathbf{1 8}$ & $\mathbf{3}$ & 99 & $15^{1} 17^{4} 15^{1}$ \\
$\mathbf{1 9}$ & $\mathbf{3}$ & 109 & $6^{1}$ examples \\
$\mathbf{2 0}$ & $\mathbf{3}$ & 111 & 14 examples \\
$\mathbf{2 1}$ & $\mathbf{3}$ & 117 & $18^{1} 20^{4} 18^{1}$
\end{tabular}

of that table obtained using our own program. Results not included in the original table are indicated in bold; if the $k$ and $i$ values are bolded then the row did not appear in the original at all. A horizontal line separates the entries with $k<2 i$ from the entries with $k \geq 2 i$. The fact that $Q(13-5,5)=115$ is of particular interest; this is the easiest-to-compute value for which Landman's conjecture fails.

A natural direction for further study is to try to obtain upper bounds similar to Theorem 1.11 for quasi-progressions with smaller diameter. While it seems unlikely that exact results can be obtained, it may be possible to get the correct order of growth. Landman proved $Q(k-1, k)=2 k-1$ and Theorem 1.11 says in essence that $Q\left(\frac{k}{2}, k\right) \leq 2 k^{2}$. Accordingly, we make the following conjecture.

Conjecture 1.15. For a fixed positive integer $r$, there exists a constant $c$ such that

$$
Q\left(\frac{k}{r}, k\right) \leq c k^{r}
$$


Table 1.2: Verified and updated version of the table from [8] $(i=4)$

\begin{tabular}{rrll}
\hline$k$ & $i$ & $Q(k-i, k)$ & \multicolumn{1}{c}{ Maximal Valid Colorings } \\
\hline \hline 4 & 4 & 35 & $\mathbf{1 4}$ examples \\
5 & 4 & 33 & $4^{8}$ and $\mathbf{4 3}$ others \\
6 & 4 & 49 & $5^{4} 4^{2} 5^{4}$ and $\mathbf{8}$ others \\
7 & 4 & 65 & $6^{2} 4^{1} 6^{2} 4^{1} 6^{2} 4^{1} 6^{2} 4^{1}, 6^{1} 4^{1} 6^{2} 4^{1} 6^{2} 4^{1} 6^{2} 4^{1} 6^{1}, 4^{1} 6^{2} 4^{1} 6^{2} 4^{1} 6^{2} 4^{1} 6^{2}$ \\
\hline 8 & 4 & 51 & $4^{1} 7^{6} 4^{1}$ and $\mathbf{6}$ others \\
9 & 4 & 65 & $8^{8}, 4^{1} 8^{7} 4^{1}$ \\
10 & 4 & 67 & $8^{3} 9^{2} 8^{3}$ and $\mathbf{1 4}$ others \\
11 & 4 & 75 & $\mathbf{6}$ examples \\
12 & 4 & 83 & $8^{1} 11^{6} 8^{1}$ and $\mathbf{6}$ others \\
13 & 4 & 97 & $12^{8}, 8^{1} 12^{7} 4^{1}, 4^{1} 12^{7} 8^{1}$ \\
14 & 4 & 99 & $12^{3} 13^{2} 12^{3}$ and 20 others \\
$\mathbf{1 5}$ & $\mathbf{4}$ & 107 & 9 examples \\
$\mathbf{1 6}$ & $\mathbf{4}$ & 115 & 7 examples \\
$\mathbf{1 7}$ & $\mathbf{4}$ & 129 & $16^{8}, 12^{1} 16^{7} 4^{1}, 8^{1} 16^{7} 8^{1}, 4^{1} 16^{7} 12^{1}$ \\
$\mathbf{1 8}$ & $\mathbf{4}$ & 131 & 7 examples \\
$\mathbf{1 9}$ & $\mathbf{4}$ & 139 & 12 examples
\end{tabular}


Table 1.3: Verified and updated version of the table from [8] $(i \geq 5)$

\begin{tabular}{rrrl}
\hline$k$ & $i$ & $Q(k-i, k)$ & \multicolumn{1}{c}{ Maximal Valid Colorings } \\
\hline \hline 5 & 5 & 178 & $\mathbf{9 6 8 1 2}$ examples \\
6 & 5 & 67 & $2^{2} 5^{2} 2^{2} 3^{2} 2^{2} 5^{2} 2^{2} 3^{2} 2^{2} 5^{2} 2^{2}$ \\
7 & 5 & 73 & Four examples \\
8 & 5 & 93 & $\mathbf{1 9 8}$ examples \\
9 & 5 & $\mathbf{1 1 5}$ & $\mathbf{4 4}$ examples \\
\hline 10 & 5 & 83 & $5^{1} 9^{8} 5^{1}$ and 25 others \\
11 & 5 & 101 & $10^{10}, 5^{1} 10^{9} 5^{1}$ \\
12 & 5 & $\mathbf{1 0 3}$ & $\mathbf{1 0}$ examples \\
13 & 5 & $\mathbf{1 1 5}$ & $5^{1} 10^{4} 12^{2} 10^{4} 5^{1}$ \\
14 & 5 & $\mathbf{1 2 3}$ & $5^{1} 10^{2} 13^{2} 10^{2} 13^{2} 10^{2} 5^{1}$ \\
15 & 5 & $\mathbf{1 3 3}$ & $\mathbf{2 6}$ examples \\
$\mathbf{1 6}$ & $\mathbf{5}$ & 151 & $15^{10}, 10^{1} 15^{9} 5^{1}, 5^{1} 15^{9} 10^{1}$ \\
$\mathbf{1 7}$ & $\mathbf{5}$ & 153 & 40 examples \\
& & & \\
7 & 6 & $\mathbf{1 2 7}$ & 52256466664255246511566432362141 \\
& & & 522564666642552466666432362141 \\
$\mathbf{1 1}$ & $\mathbf{6}$ & 184 & 94 examples $(\mathrm{see} Q P \operatorname{Qirc})$ \\
\hline $\mathbf{1 2}$ & $\mathbf{6}$ & 123 & 61 examples \\
$\mathbf{1 5}$ & $\mathbf{6}$ & $\geq 161$ & $6^{1} 12^{5} 14^{2} 12^{5} 6^{1}$ \\
& & & \\
8 & 7 & $\geq \mathbf{1 9 4}$ & $7263634353377422552562575-$ \\
& & & -12267472256226572133351 \\
9 & 8 & $\mathbf{2 8 9}$ & $82836386822482622723782342252472-$ \\
& & & -88578852484422168253442387428231
\end{tabular}




\section{CHAPTER 2}

\section{PRETENDING: THE ROW SPACE OF AN ADJACENCY MATRIX}

At the 21st British Combinatorial Conference, Peter Cameron posed this question:

Question 2.1 (Cameron, [4]). Let $G$ be a nonempty graph and let $A$ be the adjacency matrix of $G$. Is there always a nonzero $\{0,1\}$-vector in Row $A$ (over $\mathbb{R}$ ) that is not a row of $A$ ?

While Cameron posed the question for vectors over the real numbers, $\mathbb{R}$, note that it suffices to work over the rational numbers, $\mathbb{Q}$.

\section{1 - Introduction}

For basic linear algebra terminology, notation, and results, see [10]. For basic graph theory, see [14]. Recall the definition of the adjacency matrix of a graph.

Definition 2.2. Let $G$ be a graph with vertex set $V(G)=\left\{v_{1}, v_{2}, \ldots, v_{n}\right\}$. The adjacency matrix of $G$, denoted $A(G)$, is the $n \times n\{0,1\}$-matrix with $a_{i j}=1$ if and only if $v_{i}$ and $v_{j}$ are adjacent in $G$.

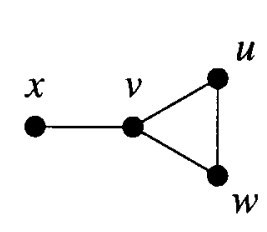

$$
A(G)=\left[\begin{array}{llll}
u & v & w & x \\
0 & 1 & 1 & 0 \\
1 & 0 & 1 & 1 \\
1 & 1 & 0 & 0 \\
0 & 1 & 0 & 0
\end{array}\right]
$$

Definition 2.3. The rank of a graph $G$ is the rank of $A(G)$ (over $\mathbb{R}$ ).

In their unpublished manuscript [1], Akbari, Cameron, and Khosrovshahi noticed that many graph parameters have bounds related to the rank of the graph.

Proposition 2.4 ([1, p. 10-13]). Let $G$ be a graph with rank $r$. Each of these graph parameters is bounded by a function of $r$.

- The number of connected components (aside from isolated vertices) is at most $\left\lfloor\frac{r}{2}\right\rfloor$ 
with equality if and only if at most one component is complete tripartite and the rest are complete bipartite.

- The clique number $\omega(G) \leq r$, with equality if and only if $G$ is a complete $r$-partite graph (possibly with isolated vertices).

- The chromatic number $\chi(G)$ is bounded by some function of $r$, but Raz and Spieker [11] have shown that $\chi(G)$ is not bounded by any polynomial function of $r$.

- For fixed $k$, the smallest number of factors in an edge partition into complete $k$-partite graphs is bounded by an unspecified function of $r$.

- If $G$ has no isolated vertices, then the domination number $\gamma(G) \leq r$ with equality if and only if $G$ is a complete bipartite graph $K_{k, l}$ with $k, l \geq 2$.

- If $G$ has no isolated vertices, then the total domination number $\gamma_{t}(G) \leq r$ with equality if and only if each component of $G$ is complete bipartite.

- If $G$ is connected, then the diameter $\operatorname{diam} G \leq r$.

- The order of the largest composition factor of the group $\operatorname{Aut}(G)$ which is not an alternating group is bounded by $r$ but not by a polynomial function of $r$.

They are able to say more, but first we need some definitions.

Definition 2.5. Let $G$ be a graph and let $u, v \in V(G)$. If $N(u)=N(v)$ then we say that $u$ and $v$ are twins in $G$.

Isolated vertices will induce $\overrightarrow{\mathbf{0}}$ as a row of $A(G)$. Twin vertices will produce two copies of the same row in $A(G)$. Therefore adding or deleting isolated vertices or twin vertices will not affect the rank of $G$.

Definition 2.6. A simple graph $G$ is said to be a reduced graph if and only if $G$ has no isolated vertices and $G$ has no twins.

Akbari, Cameron, and Khosrovshahi prove that there are only finitely many reduced graphs with a given rank. They give a lower bound on $m(r)$, the largest order of a reduced 
graph with rank $r$ :

$$
m(r) \geq \begin{cases}2^{(r+2) / 2}-1 & \text { if } r \text { is even } \\ 5 \cdot 2^{(r-3) / 2}-1 & \text { if } r \text { is odd and } r>1\end{cases}
$$

and conjecture that their bound is actually the correct value. If every graph has a vector as described in question 2.1 , then $m(r)$ is an increasing function. Thus we will reformulate question 2.1 as a conjecture.

Conjecture 2.7. If $G$ is a reduced graph then $\operatorname{Row} A(G)$ contains a nonzero $\{0,1\}$-vector that is not a row of the matrix.

Definition 2.8 (hood vector). Let $M$ be a $\{0,1\}$-matrix and let $\overrightarrow{\mathbf{v}} \in$ Row $M$ be nonzero. We say $\overrightarrow{\mathbf{v}}$ is a hood vector if $\overrightarrow{\mathbf{v}}$ is a $\{0,1\}$-vector and $\overrightarrow{\mathbf{v}}$ is not a row of $M$. If $M=A(G)$ for some graph $G$, we also say that $\overrightarrow{\mathbf{v}}$ is a hood vector of $G$.

Rephrased in this language, Conjecture 2.7 says that every reduced graph has a hood vector. A hood vector of a graph 'looks like' the rows of $A(G)$, which correspond to the neighborhoods of vertices of $G$. Thus the hood vector 'looks like' a neighborhood in $G$.

Definition 2.9. Let $G$ be a graph with a hood vector $\overrightarrow{\mathbf{v}}$. The shadow neighborhood of $\overrightarrow{\mathbf{v}}$ in $G$ is the set of vertices whose corresponding position in $\overrightarrow{\mathbf{v}}$ is equal to 1 .

Definition 2.10. Let $v$ be a vertex of a graph $G$. The neighborhood vector of $v$, denoted $N(\overrightarrow{v)}$, is the row of $A(G)$ corresponding to $v$.

\section{2 - Basic Results}

Any graph whose adjacency matrix has full rank will have $\overrightarrow{\mathbf{1}}$ as a hood vector. Costello and $\mathrm{Vu}$ [5] have shown that the adjacency matrix of a random graph will almost surely have full rank. This suggests that a probabilistic approach will not yield the result. It may be fruitful to find some properties that any counterexample to Conjecture 2.7 must satisfy. Lemma 2.11. In any counterexample $G$ to Conjecture 2.7 , every edge of $G$ must lie on a triangle. 
Proof. Assume $G$ has an edge $u v$ not on a triangle. This implies $N(u) \cap N(v)=\varnothing$. Therefore $N \overrightarrow{(u)}+N \overrightarrow{(v)}$ is a $\{0,1\}$-vector. Any vertex with this vector as its neighborhood would have to be adjacent to both $u$ and $v$, which is impossible. Thus, the vector produced is a hood vector.

Proposition 2.12. Let $G$ be a counterexample to Conjecture 2.7. If $u$ and $v$ are vertices of $G$ with disjoint neighborhoods, then there exists a vertex $w$ such that $N(w)=N(u) \cup N(v)$. Proof. The vector $N \overrightarrow{(u)}+N \overrightarrow{(v)}$ is a $\{0,1\}$-vector that is 1 exactly in the positions corresponding to $N(u) \cup N(v)$.

Corollary 2.13. If $G$ is a counterexample to Conjecture 2.7 then diam $G \leq 4$.

Proposition 2.14. If $G$ is a counterexample to Conjecture 2.7 then $\operatorname{diam} G \leq 3$.

Proof. Corollary 2.13 tells us that $\operatorname{diam} G \leq 4$, so assume diam $G=4$ to obtain a contradiction. Let $u$ and $v$ be vertices of $G$ such that $d(u, v)=4$. Not only are the neighborhoods of $u$ and $v$ disjoint, we also know that no edges run between these neighborhoods. Let $w \in N(v)$.

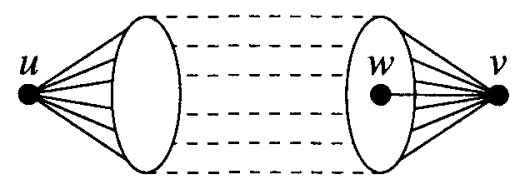

The vector $N \overrightarrow{(u)}+N \overrightarrow{(w)}$ is a $\{0,1\}$-vector since no edges run between $N(u)$ and $N(v)$. This vector is 1 on $v$, all vertices in $N(u)$, and possibly on some other vertices of no consequence. If this vector were the neighborhood vector of a vertex, that vertex would have to be in $N(v)$ and adjacent to vertices in $N(u)$, which is impossible.

Lemma 2.15. In any counterexample $G$ to Conjecture 2.7 , every triangle of $G$ must be contained in a $K_{4}$ or an induced lolliop $L_{3,1}$.

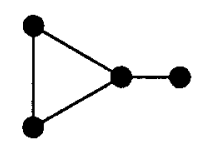

\section{Lollipop $L_{3,1}$}

Proof. To obtain a contradiction assume that $u, v$, and $w$ are vertices in $G$ of a triangle not contained in any $K_{4}$ or $L_{3,1}$. Thus every vertex of $G$ is adjacent to exactly 0 or 2 of $u, v$, and 
$w$. Therefore the vector $\frac{1}{2} N \overrightarrow{(u)}+\frac{1}{2} N\left(\overrightarrow{v)}+\frac{1}{2} N \overrightarrow{(w)}\right.$ is a $\{0,1\}$-vector. This vector describes a neighborhood containing $u, v$, and $w$, which implies the vector is a hood vector since no vertex in $G$ is adjacent to all three of $u, v$, and $w$.

We can use Lemma 2.15 to obtain a nice result, but first we need some definitions.

Definition 2.16 (From [14, p. 281]). Let $G$ be a simple graph.

A triangle $T$ is odd if and only if some vertex of $G$ is adjacent to an odd number of vertices of $T$. That is, there exists $v \in V(G)$ such that $|N(v) \cap V(T)|$ is odd.

A triangle $T$ is even if and only if every vertex of $G$ is adjacent to an even number of vertices of $T$. That is, for all $v \in V(G)$ we have $|N(v) \cap V(T)|$ is even.

Thus Lemma 2.15 says that in a counterexample, every triangle must be odd. Cameron stated that Conjecture 2.7 is true for line graphs. We can now prove this result using this characterization of line graphs ([12], paraphrased from [14, p. 281]):

Theorem 2.17 (van Rooij and Wilf, 1965). The graph $G$ is a line graph if and only if $G$ is claw-free and no induced diamond of $G$ has two odd triangles.

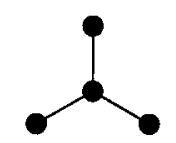

Claw $K_{1,3}$

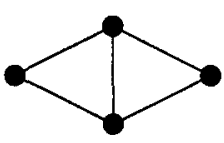

Diamond $K_{4}-e$

Theorem 2.18. Every line graph has a hood vector.

Proof. Let $G$ be a line graph. If $G$ has an induced diamond, then by Theorem 2.17 at least one of the triangles must be even and so $G$ must have a hood vector. If $G$ does not contain an induced diamond then $G$ is claw-free. Every edge of $G$ must be on a triangle by Lemma 2.11 .

Take a maximal clique $C$ in $G$. Since every edge of $G$ is on a triangle, $C$ must have at least three vertices. If $C=G$ then $A(G)$ has full rank and so $\overrightarrow{\mathbf{1}}$ is a hood vector. Otherwise, choose a vertex $v$ of $G$ which is adjacent to some $u \in V(C)$ but is not itself in $C$. Since $C$ is maximal, there is some $w \in V(C)$ such that $v$ is not adjacent to $w$. In fact $v$ cannot be adjacent to any vertex in $C$ except $u$, since if there were some $x \in V(C)$ adjacent to $v$, $\{u, x, w, v\}$ would induce a diamond. 


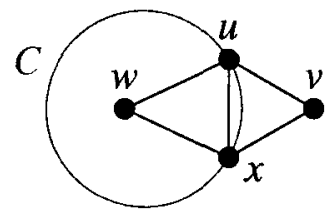

Thus, $G$ is composed of maximal cliques (each containing at least three vertices) joined at single vertices. Furthermore, since $G$ is claw-free no vertex can be in more than two such cliques. Call this collection of maximal cliques $\mathcal{M}$. We divide into cases based on $|\mathcal{M}|$.

Case 1. If $|\mathcal{M}|=1$ then $G$ is a complete graph, a situation previously handled.

Case 2. If $|\mathcal{M}|=2$ then $G$ is a pair of cliques $K_{k}$ and $K_{l}$ joined at a vertex $v$. We can obtain $\overrightarrow{\mathbf{1}}$ as a hood vector $G$ using the linear combination

$$
\overrightarrow{\mathbf{1}}=\sum_{x \in K_{k} \backslash\{v\}} a N \overrightarrow{(x)}+b N \overrightarrow{(v)}+\sum_{x \in K_{i} \backslash\{v\}} c N \overrightarrow{(x)}
$$

where $a, b, c$ satisfy

$$
(k-2) a+b=1 \quad(k-1) a+(l-1) c=1 \quad b+(l-2) c=1
$$

This linear system has the solution

$$
a=\frac{2-l}{3 l+3 k-2 k l-4} \quad b=\frac{l+k-k l}{3 l+3 k-2 k l-4} \quad c=\frac{2-k}{3 l+3 k-2 k l-4}
$$

To see that this solution is always valid, assume $3 l+3 k-2 k l-4=0$. Then

$$
l=\frac{3 k-4}{2 k-3}=\frac{2 k-3+k-1}{2 k-3}=1+\frac{k-1}{2 k-3}
$$

Since every clique in $\mathcal{M}$ has at least three vertices, $k \geq 3$ and

$$
(2 k-3)-(k-1)=k-2 \geq 3-2=1>0
$$

Therefore $2 k-3>k-1$. But then equation 2.1 forces $1<l<2$, which cannot possibly be a valid value for $l$. Thus the solution to this linear system is always valid.

Case 3. If $|\mathcal{M}| \geq 3$, there are two possibilities.

Case 3.1. First, suppose $\mathcal{M}$ contains three cliques $X, Y, Z$ which all pairwise intersect. They do so at distinct vertices $v_{X Y}, v_{X Z}$, and $v_{Y Z}$. Let $z \in Z \backslash\left\{v_{X Z}, v_{Y Z}\right\}$. This means $z$ is adjacent to $v_{X Z}$ and $v_{Y Z}$ but not $v_{X Y}$. Therefore $\left\{v_{X Y}, v_{X Z}, v_{Y Z}\right\}$ induces a diamond, contradicting our assumptions. 


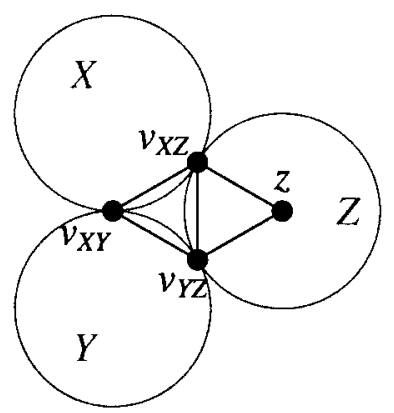

Case 3.2. On the other hand if $\mathcal{M}$ has no triple that all pairwise intersect, then taking $X, Y, Z \in \mathcal{M}$, we can assume without loss of generality that $X$ and $Z$ do not intersect. If there exist $x \in X$ and $z \in Z$ such that $x$ is not in a clique containing an element of $Z$ and $z$ is not in a clique containing an element of $X$, then the $\{0,1\}$-vector $N \overrightarrow{(x)}+N \overrightarrow{(z)}$ is a hood vector.

If such elements do not exist, then (without loss of generality) every element of $X$ shares a clique with an element of $Z$. Let $x_{1}, x_{2}, x_{3} \in X$. There exist $z_{1}, z_{2}, z_{3} \in Z$ and $Y_{1}, Y_{2}, Y_{3} \in \mathcal{M}$ such that $x_{i}, z_{i} \in V\left(Y_{i}\right)$. Since $\mathcal{M}$ has no triple that all pairwise intersect, two of $\left\{Y_{1}, Y_{2}, Y_{3}\right\}$ must not intersect; without loss of generality we will assume $Y_{1}$ and $Y_{2}$ do not intersect. Then the $\{0,1\}$-vector $\frac{1}{2} N \overrightarrow{\left(x_{1}\right)}+\frac{1}{2} N \overrightarrow{\left(x_{2}\right)}+\frac{1}{2} N \overrightarrow{\left(z_{1}\right)}+\frac{1}{2} N \overrightarrow{\left(z_{2}\right)}$ is a hood vector.

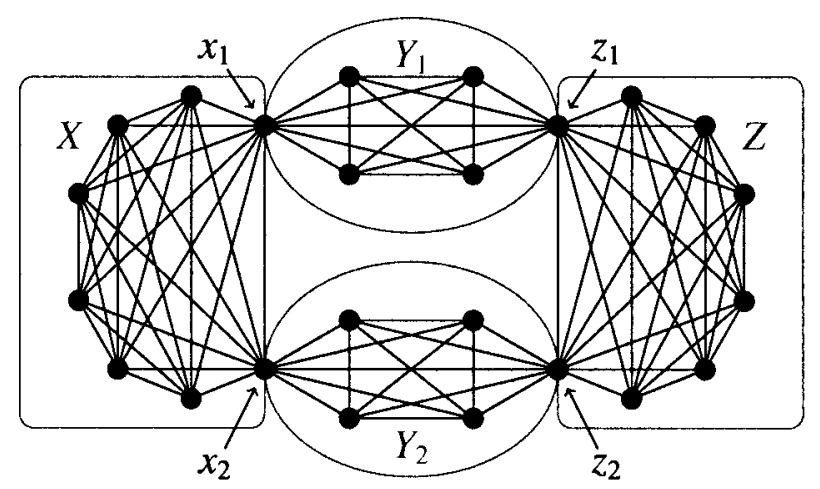

All the cases are accounted for and thus we have the result.

One might be tempted to try to build a counterexample to Conjecture 2.7 by starting with some base graph and appending vertices corresponding to hood vectors in hopes of extinguishing the list. This next proposition shows that such an approach is unlikely to succeed. 
Proposition 2.19. Let $G$ be a graph with a hood vector $\overrightarrow{\mathbf{x}}$. Let

$$
\overrightarrow{\mathbf{x}}=c_{1} N \overrightarrow{\left(v_{1}\right)}+\cdots+c_{k} N \overrightarrow{\left(v_{k}\right)}+c_{k+1} N \overrightarrow{\left(v_{k+1}\right)}+\cdots+c_{n} N \overrightarrow{\left(v_{n}\right)}
$$

where $v_{1}, \ldots, v_{k}$ are the vertices in the shadow neighborhood of $\overrightarrow{\mathbf{x}}$ and $v_{k+1}, \ldots, v_{n}$ are the other vertices of $G$. Let $G^{\prime}$ be the graph obtained from $G$ by appending the vertex with neighborhood vector $\overrightarrow{\mathbf{x}}$. If $c_{1}+\cdots+c_{k} \neq 0$ then $G^{\prime}$ also contains a hood vector.

Proof. Append the vertex $v_{n+1}$ with neighborhood $v_{1}, v_{2}, \ldots, v_{k}$ and let

$$
\Gamma=c_{1}+c_{2}+\cdots+c_{k} \neq 0
$$

We can obtain a new hood vector $\overrightarrow{\mathbf{y}}=\left\langle y_{1}, \ldots, y_{n}\right\rangle$ by letting

$$
\overrightarrow{\mathbf{y}}=\frac{c_{1}}{\Gamma} N \overrightarrow{\left(v_{1}\right)}+\cdots+\frac{c_{n}}{\Gamma} N\left(\overrightarrow{\left.v_{n}\right)}+\frac{\Gamma-1}{\Gamma} N \overrightarrow{\left(v_{n+1}\right)}\right.
$$

which will produce

$$
y_{1}=y_{2}=\cdots=y_{k}=y_{n+1}=1 \quad y_{k+1}=\cdots=y_{n}=0
$$

which cannot be the neighborhood vector of any vertex since no vertex in $G$ had neighborhood $v_{1}, v_{2}, \ldots, v_{k}$.

\section{3 - Generalization}

What is the adjacency matrix of a graph? Ultimately, it is just a symmetric $\{0,1\}$-matrix with 0 on the main diagonal. If Conjecture 2.7 is true, then it seems reasonable to ask whether each of these conditions is necessary. Perhaps Conjecture 2.7 follows as a corollary to a more general statement.

Question 2.20. Does every $\{0,1\}$-matrix have a hood vector?

The answer is no, as this example shows.

Example 2.21 ( $\{0,1\}$-matrix with no hood vectors). The matrix $M$ has no hood vectors.

$$
M=\left[\begin{array}{lllllll}
0 & 1 & 1 & 0 & 0 & 1 & 1 \\
1 & 1 & 0 & 1 & 1 & 0 & 0 \\
1 & 0 & 1 & 0 & 1 & 1 & 1 \\
1 & 1 & 0 & 1 & 0 & 1 & 1 \\
0 & 1 & 1 & 0 & 1 & 0 & 0 \\
1 & 0 & 1 & 1 & 0 & 0 & 1 \\
1 & 0 & 1 & 1 & 0 & 1 & 0
\end{array}\right]
$$


Proof. In reduced row echelon form $M$ is

$$
\left[\begin{array}{ccccccc}
1 & 0 & 0 & 0 & 0 & 0 & \frac{7}{2} \\
0 & 1 & 0 & 0 & 0 & 0 & \frac{3}{2} \\
0 & 0 & 1 & 0 & 0 & 0 & \frac{1}{2} \\
0 & 0 & 0 & 1 & 0 & 0 & -3 \\
0 & 0 & 0 & 0 & 1 & 0 & -2 \\
0 & 0 & 0 & 0 & 0 & 1 & -1 \\
0 & 0 & 0 & 0 & 0 & 0 & 0
\end{array}\right]
$$

Thus rank $M=6$ and $\overrightarrow{\mathbf{n}}=\langle 7,3,1,-6,-4,-2,-2\rangle \in \operatorname{Nul} M$. Moreover $\operatorname{Span}\{\overrightarrow{\mathbf{n}}\}=$ Nul $M$, so vectors in Nul $M$ have the form $k \cdot \overrightarrow{\mathbf{n}}$ for some $k \in \mathbb{R}$. Suppose $\overrightarrow{\mathbf{a}}=\left\langle a_{i}\right\rangle \in$ Row $M$. Then

$$
\overrightarrow{\mathbf{a}} \cdot \overrightarrow{\mathbf{n}}=7 a_{1}+3 a_{2}+a_{3}+{ }^{-} 6 a_{4}+-4 a_{5}+{ }^{-} 2 a_{6}+{ }^{-} 2 a_{7}=0
$$

and so

$$
7 a_{1}+3 a_{2}+a_{3}=6 a_{4}+4 a_{5}+2 a_{6}+2 a_{7}
$$

If $\overrightarrow{\mathbf{a}}$ is a non-zero $\{0,1\}$-vector, then since all the left-hand coefficients are odd and all the right-hand coefficients are even, $\overrightarrow{\mathbf{a}}$ must have a 1 in exactly two of the first three positions. Therefore the following solutions are the only possible.

$$
\begin{array}{ll}
7+3+0=6+4+0+0 & \langle 1,1,0,1,1,0,0\rangle=\overrightarrow{\mathbf{r}_{2}} \\
7+3+0=6+0+2+2 & \langle 1,1,0,1,0,1,1\rangle=\overrightarrow{\mathbf{r}_{4}} \\
7+0+1=6+0+2+0 & \langle 1,0,1,1,0,1,0\rangle=\overrightarrow{\mathbf{r}_{7}} \\
7+0+1=6+0+0+2 & \langle 1,0,1,1,0,0,1\rangle=\overrightarrow{\mathbf{r}_{6}} \\
7+0+1=0+4+2+2 & \langle 1,0,1,0,1,1,1\rangle=\overrightarrow{\mathbf{r}_{3}} \\
0+3+1=0+4+0+0 & \langle 0,1,1,0,1,0,0\rangle=\overrightarrow{\mathbf{r}_{5}} \\
0+3+1=0+0+2+2 & \langle 0,1,1,0,0,1,1\rangle=\overrightarrow{\mathbf{r}_{1}}
\end{array}
$$

Thus $\overrightarrow{\mathbf{a}}$ must be a row of $M$, and so $M$ has no hood vectors. 
Note that $M$ is not symmetric and has some 1s on the main diagonal. Further, $M^{T}$ has forty hood vectors. Thus it seems unlikely that a simple modification to $M$ will produce a counterexample to Conjecture 2.7 .

$$
M=\left[\begin{array}{cccccccc}
0 & 1 & 1 & 0 & 0 & 1 & 1 \\
1 & 1 & 0 & 1 & 1 & 0 & 0 \\
1 & 0 & 1 & 0 & 1 & 1 & 1 \\
1 & 1 & 0 & 1 & 0 & 1 & 1 \\
\hdashline 0 & 1 & 1 & 0 & 1 & 0 & 0 \\
1 & 0 & 1 & 1 & 0 & 0 & 1 \\
1 & 0 & 1 & 1 & 0 & 1 & 0
\end{array}\right]
$$

That said, example 2.21 generalizes to an entire family of $\{0,1\}$-matrices without hood vectors.

Example 2.22. Let $m$ be even. Then the matrix $M$ has no hood vectors.

$$
M=\left[\begin{array}{ccccccccc}
0 & 1 & 1 & 0 & 0 & \overbrace{1} & 1 & \ldots & 1 \\
1 & 1 & 0 & 1 & 1 & 0 & 0 & \ldots & 0 \\
1 & 0 & 1 & 0 & 1 & 1 & 1 & \ldots & 1 \\
1 & 1 & 0 & 1 & 0 & 1 & 1 & \ldots & 1 \\
0 & 1 & 1 & 0 & 1 & 0 & 0 & \ldots & 0 \\
\hline 1 & 0 & 1 & 1 & 0 & 0 & 1 & \ldots & 1 \\
1 & 0 & 1 & 1 & 0 & 1 & 0 & & 1 \\
& & & \vdots & & \vdots & & \ddots & \\
1 & 0 & 1 & 1 & 0 & 1 & 1 & \ldots & 0
\end{array}\right]
$$

Proof. We will construct a vector $\overrightarrow{\mathbf{n}} \in \operatorname{Nul} M$ that is orthogonal only to non-zero $\{0,1\}$ vectors that are rows of $M$. Let $\overrightarrow{\mathbf{n}}=\langle a, b, c, x, y, w, w, \ldots, w\rangle$. Then if $\overrightarrow{\mathbf{n}} \in \operatorname{Nul} M$,

$$
\begin{aligned}
& b+c+m w=0 \quad a+b+x+m w=0 \\
& a+b+x+y=0 \quad b+c+y=0 \\
& a+c+y+m w=0 \quad a+c+x+(m-1) w=0
\end{aligned}
$$

Thus

$$
y=m w \quad x=(m+1) w
$$


And so

$$
\begin{aligned}
& a+b+(2 m+1) w=0 \quad a={ }^{-}(3 m+1) w / 2 \\
& a+c+2 m w=0 \quad \Longrightarrow \quad b=-(m+1) w / 2 \\
& b+c+m w=0 \quad c={ }^{-}(m-1) w / 2
\end{aligned}
$$

Let $w=-2$. Then $\overrightarrow{\mathbf{n}}=\langle 3 m+1, m+1, m-1,-2 m,-2 m-2,-2,-2, \ldots,-2\rangle$. By

construction $\overrightarrow{\mathbf{n}} \in \operatorname{Nul} M$. On the other hand, if $\overrightarrow{\mathbf{v}} \cdot \overrightarrow{\mathbf{n}}=0$ for some non-zero $\{0,1\}$-vector $\overrightarrow{\mathbf{v}}$, then exactly two of the first three entries in $\overrightarrow{\mathbf{v}}$ must be 1 , and all possible ways to balance such a sum are already present in $M$.

Each member of this infinite family of examples has one asymmetry and four non-zero values on the main diagonal. However, the transposes of these matrices contain many hood vectors. Example 2.32 will show another family of $\{0,1\}$-matrices that avoid hood vectors in the matrix as well as the transpose, but that have a non-constant number of asymmetries and non-zero entries on the main diagonal. Furthermore, example 2.33 shows that there is a family of symmetric $\left\{0, \frac{1}{2}, 1\right\}$-matrices without hood vectors.

These examples show that if Conjecture 2.7 is true then it is close to the best possible statement. It is hard to imagine that the main diagonal entries are of actual importance, so it is reasonable to strengthen Conjecture 2.7 by removing this requirement.

Conjecture 2.23. Every symmetric $\{0,1\}$-matrix has a hood vector.

\section{4 - Searching and Verification}

This section investigates algorithmic aspects of the problem of finding hood vectors. By expressing the problem of finding a hood vector as an integer linear program we can enlist powerful computer libraries and toolkits to assist us in our search for a counterexample. We also develop a short certificate that allows us to quickly count the number of hood vectors without explicitly listing them. Linear algebra provides the framework for both aspects. Recall the following well-known theorem: 
Theorem 2.24. Given a basis $\overrightarrow{\mathbf{n}_{1}}, \ldots, \overrightarrow{\mathbf{n}_{k}}$ for Nul $M$, a vector $\overrightarrow{\mathbf{x}} \in$ Row $M$ if and only if $\overrightarrow{\mathbf{x}} \cdot \overrightarrow{\mathbf{n}_{i}}$ for all $i \in[k]$.

Thus, if we have a basis for $\operatorname{Nul} M$ we can easily check if a given vector is in Row $M$. That test will become the linear program. We will approach the certification problem by producing a substitute for the basis, called a monopole.

\section{Linear Programming}

Let $\overrightarrow{\mathbf{r}_{1}}, \ldots, \overrightarrow{\mathbf{r}_{n}}$ be the rows of the matrix $M$, let $d_{i}$ be the weight of row $i$, and let $\overrightarrow{\mathbf{n}_{1}}, \ldots, \overrightarrow{\mathbf{n}_{k}}$ be a basis for Nul $M$. Suppose we are given a $\{0,1\}$-vector $\overrightarrow{\mathbf{x}}$ and we want to check if it is a hood vector of $M$.

To ensure $\overrightarrow{\mathbf{x}}$ is not already a row of our matrix, we can check that it differs in some position from each $\overrightarrow{\mathbf{r}_{i}}$. Since $\overrightarrow{\mathbf{x}}$ and $\overrightarrow{\mathbf{r}_{i}}$ are both $\{0,1\}$-vectors, $\overrightarrow{\mathbf{x}} \cdot \overrightarrow{\mathbf{r}_{i}}$ will count the number of positions in which $\overrightarrow{\mathbf{x}}$ and $\overrightarrow{\mathbf{r}_{i}}$ are both 1 . Similarly $(\overrightarrow{\mathbf{1}}-\overrightarrow{\mathbf{x}}) \cdot\left(\overrightarrow{\mathbf{1}}-\overrightarrow{\mathbf{r}_{i}}\right)$ will count the number of positions in which $\overrightarrow{\mathbf{x}}$ and $\overrightarrow{\mathbf{r}_{i}}$ are both 0 . Thus

$$
\begin{aligned}
n & \geq \overrightarrow{\mathbf{x}} \cdot \overrightarrow{\mathbf{r}_{i}}+(\overrightarrow{\mathbf{1}}-\overrightarrow{\mathbf{x}}) \cdot\left(\overrightarrow{\mathbf{1}}-\overrightarrow{\mathbf{r}_{i}}\right) \\
& =\sum_{j=1}^{n} x_{j} \cdot r_{i j}+\sum_{j=1}^{n}\left(1-x_{j}\right) \cdot\left(1-r_{i j}\right) \\
& =\sum_{j=1}^{n} x_{j} \cdot r_{i j}+1-x_{j}-r_{i j}+x_{j} \cdot r_{i j} \\
& =\sum_{j=1}^{n}\left(2 x_{j} \cdot r_{i j}-x_{j}\right)+\sum_{j=1}^{n} 1-\sum_{j=1}^{n} r_{i j} \\
& =\sum_{j=1}^{n}\left(2 x_{j} \cdot r_{i j}-x_{j}\right)+n-d_{i}
\end{aligned}
$$

and therefore

$$
d_{i} \geq \sum_{j=1}^{n} x_{j}\left(2 r_{i j}-1\right)
$$

where equality holds if and only if $\overrightarrow{\mathbf{x}}=\overrightarrow{\mathbf{r}_{i}}$.

With this in mind we can formulate the problem of finding a hood vector as an integer linear program: 
Maximize:

$$
\sum_{i=1}^{n} x_{i}
$$

Subject to:

$$
\begin{gathered}
\sum_{j=1}^{n} x_{j} \cdot n_{i j}=0 \text { for all } i \in[k] \\
\sum_{j=1}^{n} x_{j}\left(2 r_{i j}-1\right) \leq d_{i}-1 \text { for all } i \in[n] \\
x_{i} \in\{0,1\} \text { for all } i \in[n]
\end{gathered}
$$

This program will return a $\{0,1\}$-vector of maximum weight in Row $M$ that is not a row of $M$. Thus, if it does not return $\overrightarrow{\mathbf{0}}$ it will return a hood vector, and if it does return $\overrightarrow{0}$ then $M$ has no hood vectors.

To find the dual problem we need to first replace the equality constraint $2.2 \mathrm{a}$ with a pair of inequality constraints.

$$
\begin{gathered}
\sum_{j=1}^{n} x_{j} \cdot n_{i j} \leq 0 \text { for all } i \in[k] \\
-\sum_{j=1}^{n} x_{j} \cdot n_{i j} \leq 0 \text { for all } i \in[k]
\end{gathered}
$$

We also need to replace the integer linear program with its fractional relaxation by loosening constraint $2.2 \mathrm{c}$ :

$$
\begin{aligned}
& x_{i} \leq 1 \text { for all } i \in[n] \\
& x_{i} \geq 0 \text { for all } i \in[n]
\end{aligned}
$$

We introduce dual variables: $a_{1}^{+}, \ldots, a_{k}^{+}$for constraint $2.2 \mathrm{a}^{+} ; a_{1}^{-}, \ldots, a_{k}^{-}$for constraint $2.2 \mathrm{a}^{-} ; b_{1}, \ldots, b_{n}$ for constraint $2.2 \mathrm{~b}$; and $c_{1}, \ldots, c_{n}$ for constraint $2.2 \mathrm{c}^{\prime}$. The dual problem is given by:

Minimize:

$$
\sum_{i=1}^{n} b_{i}\left(d_{i}-1\right)+\sum_{i=1}^{n} c_{i}
$$


Subject to:

$$
\begin{aligned}
c_{j}+\sum_{i=1}^{k} n_{i j}\left(a_{i}^{+}-a_{i}^{-}\right)+\sum_{i=1}^{n} b_{i}\left(2 r_{i j}-1\right) & \geq 1 \text { for all } j \in[n] \\
a_{i}^{+}, a_{i}^{-} & \geq 0 \text { for all } i \in[k] \\
b_{i}, c_{i} & \geq 0 \text { for all } i \in[n]
\end{aligned}
$$

\section{Monopoles}

When an $n \times n$ matrix $M$ has rank $n-1$, we can use row reduction to obtain a nice integer vector $\overrightarrow{\mathbf{v}}$ such that $\operatorname{Span}\{\overrightarrow{\mathbf{v}}\}=\operatorname{Nul} M$. Thus we can check if a given $\{0,1\}$-vector is in Row $M$ by checking if it is orthogonal to $\overrightarrow{\mathbf{v}}$ and we can count how many $\{0,1\}$-vectors are in Row $M$ by counting how many subcollections of $\left\{v_{1}, \ldots, v_{n}\right\}$ sum to 0 . If $M$ has rank less than $n-1$ it is not immediately obvious that we can find a vector with the same properties as $\overrightarrow{\mathbf{v}}$ but we will establish that such a vector always exists.

Definition 2.25 (monopole). We say that $\overrightarrow{\mathbf{v}} \in \mathbb{Z}^{n}$ is a monopole for a matrix $M$ if for all $\{0,1\}$-vectors $\overrightarrow{\mathbf{x}}$,

$$
\overrightarrow{\mathbf{x}} \in \operatorname{Row} M \text { if and only if } \overrightarrow{\mathbf{x}} \cdot \overrightarrow{\mathbf{v}}=0
$$

We say $\overrightarrow{\mathbf{v}}$ is a monopole for a graph $G$ if $\overrightarrow{\mathbf{v}}$ is a monopole for $A(G)$.

Theorem 2.26. Every $\{0,1\}$-matrix has a monopole.

Proof. Let $M$ be a $\{0,1\}$-matrix with $n$ columns. If $M$ has full rank then $\overrightarrow{\mathbf{0}}$ is a monopole for $M$. Otherwise, let $\left\{\overrightarrow{\mathbf{n}_{1}}, \ldots, \overrightarrow{\mathbf{n}_{k}}\right\}$ be a basis for Nul $M$ and assume (without loss of generality) that $\overrightarrow{\mathbf{n}_{i}} \in \mathbb{Z}^{n}$ for all $i \in[k]$.

Define $m_{1}, \ldots, m_{k}$ by

$$
\begin{aligned}
m_{j} & =\max _{\overrightarrow{\mathbf{x}} \in\{0,1\}^{n}}\left\{\left|\overrightarrow{\mathbf{x}} \cdot \overrightarrow{\mathbf{n}_{j}}\right|\right\} \\
& =\max \left\{\left(\text { sum of the positive entries of } \overrightarrow{\mathbf{n}_{j}}\right), \mid \text { sum of the negative entries of } \overrightarrow{\mathbf{n}_{j}} \mid\right\}
\end{aligned}
$$

and define $c_{1}, \ldots, c_{k}$ recursively by

$$
c_{1}=1 \quad c_{j}=1+\sum_{i=1}^{j-1} c_{i} \cdot m_{i}
$$


Now let $\overrightarrow{\mathbf{v}} \in \mathbb{Z}^{n}$ be defined as

$$
\overrightarrow{\mathbf{v}}=\sum_{i=1}^{k} c_{i} \cdot \overrightarrow{\mathbf{n}_{i}}
$$

We will show that $\overrightarrow{\mathbf{v}}$ is a monopole for $M$. Let $\overrightarrow{\mathbf{x}} \in\{0,1\}^{n}$.

Assume $\overrightarrow{\mathbf{x}} \in$ Row $M$. By Theorem $2.24 \overrightarrow{\mathbf{x}} \cdot \overrightarrow{\mathbf{n}_{j}}=0$ for all $j \in[k]$.

$$
\begin{aligned}
\overrightarrow{\mathbf{x}} \cdot \overrightarrow{\mathbf{v}} & =\overrightarrow{\mathbf{x}} \cdot\left(\sum_{i=1}^{k} c_{i} \cdot \overrightarrow{\mathbf{n}_{i}}\right) \\
& =\sum_{i=1}^{k} c_{i} \cdot \overrightarrow{\mathbf{x}} \cdot \overrightarrow{\mathbf{n}_{i}} \\
& =0
\end{aligned}
$$

Now assume $\overrightarrow{\mathbf{x}} \cdot \overrightarrow{\mathbf{v}}=0$. By Theorem 2.24 it suffices to show $\overrightarrow{\mathbf{x}} \cdot \overrightarrow{\mathbf{n}_{j}}=0$ for all $j \in[k]$. Suppose not. Let $l=\max \left\{i \mid \overrightarrow{\mathbf{x}} \cdot \overrightarrow{\mathbf{n}_{i}} \neq 0\right\}$.

$$
\begin{aligned}
& 0=\overrightarrow{\mathbf{x}} \cdot \overrightarrow{\mathbf{v}}=\overrightarrow{\mathbf{x}} \cdot\left(\sum_{i=1}^{k} c_{i} \cdot \overrightarrow{\mathbf{n}_{i}}\right) \\
& 0=\sum_{i=1}^{l-1} c_{i} \cdot \overrightarrow{\mathbf{x}} \cdot \overrightarrow{\mathbf{n}_{i}}+c_{l} \cdot \overrightarrow{\mathbf{x}} \cdot \overrightarrow{\mathbf{n}_{l}}+0
\end{aligned}
$$

Therefore

$$
\begin{aligned}
c_{l} & \leq\left|c_{l} \cdot \overrightarrow{\mathbf{x}} \cdot \overrightarrow{\mathbf{n}_{l}}\right|=\left|\sum_{i=1}^{l-1} c_{i} \cdot \overrightarrow{\mathbf{x}} \cdot \overrightarrow{\mathbf{n}_{i}}\right| \\
& \leq \sum_{i=1}^{l-1} c_{i} \cdot\left|\overrightarrow{\mathbf{x}} \cdot \overrightarrow{\mathbf{n}_{i}}\right| \text { by the triangle inequality } \\
& \leq \sum_{i=1}^{l-1} c_{i} \cdot m_{i} \\
& <c_{l}
\end{aligned}
$$

a contradiction. Thus $\overrightarrow{\mathbf{x}} \cdot \overrightarrow{\mathbf{n}_{j}}=0$ for all $j \in[k]$. 
Example 2.27. Neither $M$ nor $M^{T}$ has any hood vectors.

$M=$\begin{tabular}{|ll|ll|l|ll|}
\hline 1 & 1 & $\mathbf{1}$ & $\mathbf{0}$ & 1 & 1 & 0 \\
1 & 1 & $\mathbf{0}$ & $\mathbf{1}$ & 1 & 0 & 1 \\
\cline { 1 - 3 } $\mathbf{0}$ & $\mathbf{1}$ & 0 & 0 & 0 & 1 & 0 \\
$\mathbf{1}$ & $\mathbf{0}$ & 0 & 0 & 0 & 0 & 1 \\
\cline { 1 - 2 } & 1 & 0 & 0 & 0 & 1 & 1 \\
\hline 1 & 0 & 1 & 0 & 1 & 0 & 0 \\
0 & 1 & 0 & 1 & 1 & 0 & 0 \\
\hline
\end{tabular}

Proof. In reduced row echelon form $M$ is

$$
\begin{gathered}
{\left[\begin{array}{rrrrrrr}
a & b & c & d & e & f & g \\
1 & 0 & 0 & 0 & 0 & 0 & 1 \\
0 & 1 & 0 & 0 & 0 & 1 & 0 \\
0 & 0 & 1 & 0 & 1 & 0 & -1 \\
0 & 0 & 0 & 1 & 1 & -1 & 0 \\
0 & 0 & 0 & 0 & 0 & 0 & 0 \\
0 & 0 & 0 & 0 & 0 & 0 & 0 \\
0 & 0 & 0 & 0 & 0 & 0 & 0
\end{array}\right]} \\
a=-g \\
b=-f
\end{gathered}
$$

Where $e, f, g$ are independent variables. By choosing

$$
e=3^{0}=1 \quad f=3^{1}=3 \quad g=3^{2}=9
$$

We obtain the monopole

$$
\begin{gathered}
\overrightarrow{\mathbf{n}}=\left\langle\begin{array}{rrrrrrl}
a & b & c & d & e & f & g \\
-9, & -3, & 8, & 2, & 1, & 3, & 9
\end{array}\right\rangle \\
M=\left[\begin{array}{lllllll}
1 & 1 & 1 & 0 & 1 & 1 & 0 \\
1 & 1 & 0 & 1 & 1 & 0 & 1 \\
0 & 1 & 0 & 0 & 0 & 1 & 0 \\
1 & 0 & 0 & 0 & 0 & 0 & 1 \\
1 & 1 & 0 & 0 & 0 & 1 & 1 \\
1 & 0 & 1 & 0 & 1 & 0 & 0 \\
0 & 1 & 0 & 1 & 1 & 0 & 0
\end{array}\right]
\end{gathered}
$$


It is easy to see from the construction that $\overrightarrow{\mathbf{n}}$ is orthogonal to every row of $M$. Suppose $\overrightarrow{\mathbf{a}}=\left\langle a_{i}\right\rangle$ is a $\{0,1\}$-vector orthogonal to $\overrightarrow{\mathbf{n}}$. Then

$$
\overrightarrow{\mathbf{a}} \cdot \overrightarrow{\mathbf{n}}=-9 a_{1}+-3 a_{2}+8 a_{3}+2 a_{4}+1 a_{5}+3 a_{6}+9 a_{7}=0
$$

and so

$$
8 a_{3}+2 a_{4}+1 a_{5}+3 a_{6}+9 a_{7}=9 a_{1}+3 a_{2}
$$

If we can show that equation 2.3 has exactly seven non-zero solutions then it follows that $M$ has no hood vectors. We do not count the solution where both sides sum to zero, since that represents $\overrightarrow{\mathbf{0}} \cdot \overrightarrow{\mathbf{v}}=0$. The right-hand side can sum to $0,3,9$, or 12 in exactly one way each.

\begin{tabular}{r|r}
\multicolumn{2}{c}{ Right } \\
\hline sum & ways \\
\hline 0 & 1 \\
3 & 1 \\
9 & 1 \\
12 & 1
\end{tabular}

We will construct a similar table for the left-hand side by considering each term in sequence. At first, the only possible sum is 0 . After considering 8 , we have one way to get 0 and one way to get 8 . When considering $x$, to determine the number of ways to get $i$, we just need to consider how many ways we could get $i$ or $i-x$ in the previous step. Figure 2.1 illustrates this process. After considering all the terms on the left-hand side we will have a table similar to the one we obtained for the right-hand side.

For each possible sum $i$, let $l_{i}$ be the number of ways to obtain the sum $i$ on the left and $r_{i}$ be the number of ways to obtain the sum $i$ on the right. The total number of solutions to equation 2.3 will then be

$$
\sum_{i} r_{i} \cdot l_{i}
$$

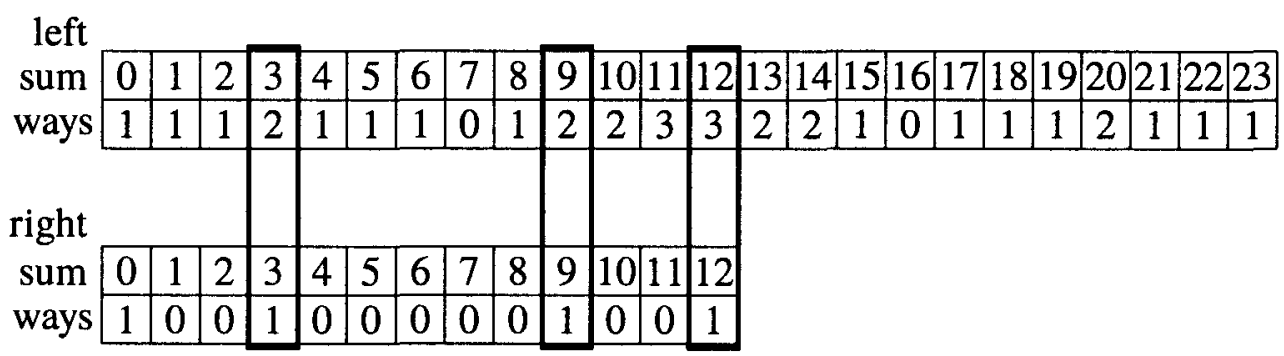




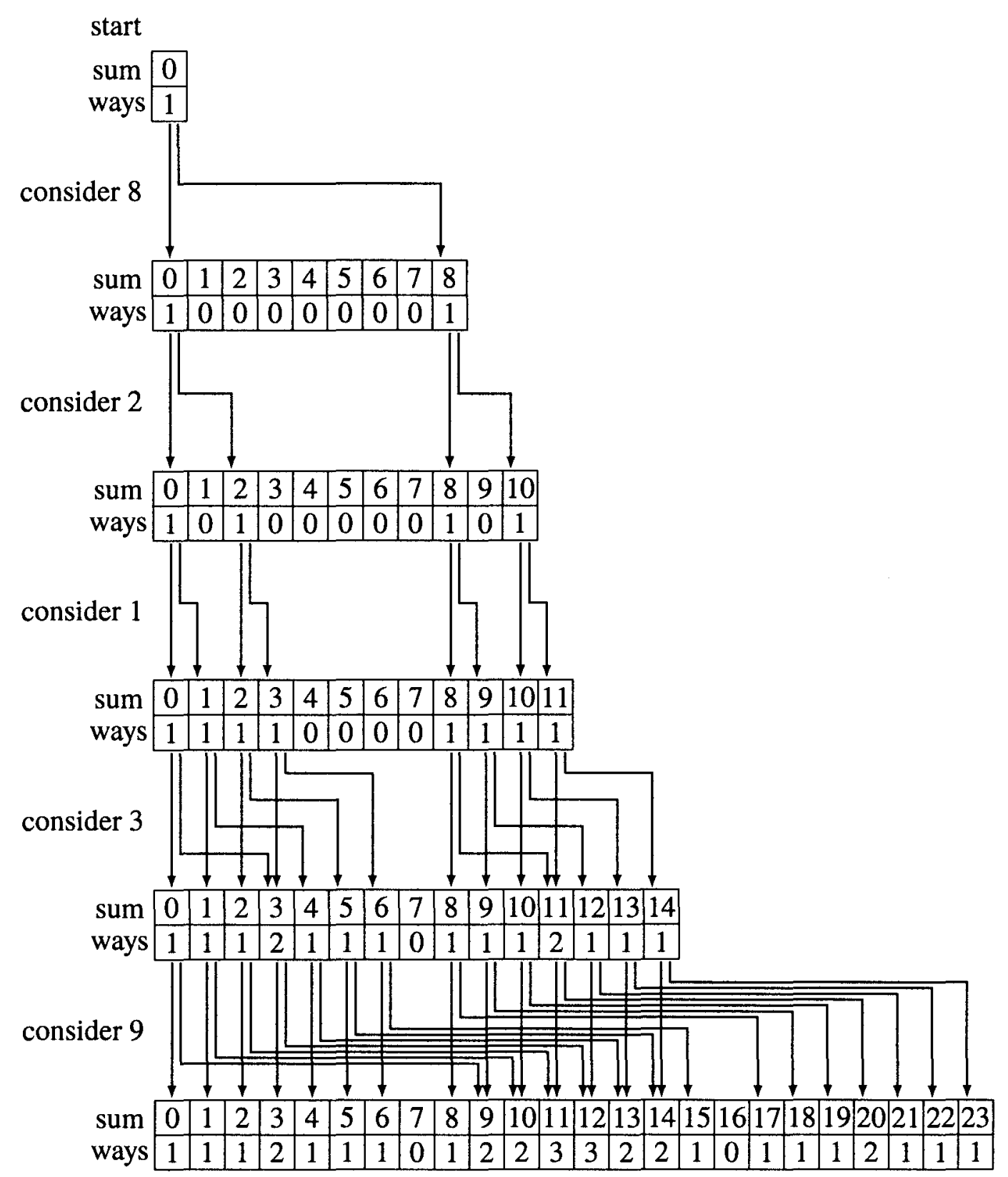

Figure 2.1: Building the table of possible sums using dynamic programming

Thus in this case the number of valid solutions is $2 \cdot 1+2 \cdot 1+3 \cdot 1=7$. Therefore $M$ has no hood vectors. The argument for $M^{T}$ is similar.

We can describe the general version of the technique we used in example 2.27 to count vectors orthogonal to the monopole, but first let us establish some simple facts about the structure of monopoles for counterexamples to Conjecture 2.7 and Conjecture 2.23. 
Lemma 2.28. Let $n \geq 2$ and let $M$ be a $n \times n$ symmetric $\{0,1\}$-matrix. If $M$ has a row of weight 1 , then $M$ has a hood vector.

Proof. To obtain a contradiction assume $M$ has no hood vectors. Let $\overrightarrow{\mathbf{v}}$ be the row of weight 1. For each row $\overrightarrow{\mathbf{u}} \neq \overrightarrow{\mathbf{v}}$, either $\overrightarrow{\mathbf{u}}+\overrightarrow{\mathbf{v}}$ or $\overrightarrow{\mathbf{u}}-\overrightarrow{\mathbf{v}}$ is also a row of $M$. Thus every column of $M$ must have weight 0 or at least 2. But $M$ has a row of weight 1, contradicting that $M$ is symmetric.

Proposition 2.29. Let $n \geq 2$ and let $M$ be an $n \times n$ symmetric $\{0,1\}$-matrix. Let $\overrightarrow{\mathbf{v}}=$ $\left\langle v_{1}, \ldots, v_{n}\right\rangle$ be a monopole for $M$. If $v_{i}=0$ for some $i \in[n]$ then $M$ has a hood vector.

Proof. Without loss of generality we may assume $v_{n}=0$. Since $\overrightarrow{\mathbf{v}} \cdot\langle 0,0, \ldots, 0,1\rangle=0, M$ has a row of weight 1. Thus $M$ has a hood vector by Lemma 2.28 .

Therefore the monopoles of any potential counterexample to Conjecture 2.7 or Conjecture 2.23 cannot have a 0 in any position.

Proposition 2.30. Let $M$ be a $\{0,1\}$-matrix with no row equal to $\overrightarrow{\mathbf{0}}$ and let $\overrightarrow{\mathbf{v}}$ be a monopole for $M$. If $\overrightarrow{\mathbf{v}}$ does not have 0 in any position and has exactly one negative (or exactly one positive) entry then $M$ has $\overrightarrow{\mathbf{1}}$ as a column.

Proof. Without loss of generality assume $v_{n}$ is the only negative entry of $\overrightarrow{\mathbf{v}}$. Let $\overrightarrow{\mathbf{r}}$ be a row of $M$. We need to show $r_{n}=1$.

$$
\begin{aligned}
r_{1} \cdot v_{1}+\cdots+r_{n-1} \cdot v_{n-1}+r_{n} \cdot v_{n} & =0 \\
r_{1} \cdot v_{1}+\cdots+r_{n-1} \cdot v_{n-1} & ={ }^{-} r_{n} \cdot v_{n}
\end{aligned}
$$

Since $\overrightarrow{\mathbf{r}} \neq \overrightarrow{\mathbf{0}}$, both sides of equation 2.4 must be nonzero. But the only way the right-hand side is nonzero is if $r_{n}=1$. Therefore the $n$th entry of every row of $M$ is a 1 and so the $n$th column of $M$ is $\overrightarrow{\mathbf{1}}$.

Since $\overrightarrow{\mathbf{1}}$ cannot be a column of the adjacency matrix of a graph, any monopole for a counterexample to Conjecture 2.7 must have at least two negative (and symmetrically, at least two positive) entries.

Returning to the problem of counting hood vectors, suppose we have a monopole $\overrightarrow{\mathbf{v}}=$ $\left\langle v_{1}, \ldots, v_{k},{ }^{-} v_{k+1}, \ldots,-{ }^{-} v_{n}\right\rangle$. Without loss of generality suppose $v_{1} \geq v_{2} \geq \cdots \geq v_{k}>0$ 
and $v_{k+1} \geq v_{k+2} \geq \cdots \geq v_{n}>0$ (recall that Proposition 2.29 guarantees $v_{i} \neq 0$ ). Given $\overrightarrow{\mathbf{a}}=\left\langle a_{i}\right\rangle$ is a $\{0,1\}$-vector orthogonal to $\overrightarrow{\mathbf{n}}$, we have

$$
v_{1} a_{1}+\cdots+v_{k} a_{k}=v_{k+1} a_{k+1}+\cdots+v_{n} a_{n}
$$

Thus the number of solutions to equation 2.5 is equal to the number of ways in which a subcollection of $\left\{v_{1}, \ldots, v_{k}\right\}$ sums to the same value as a subcollection of $\left\{v_{k+1}, \ldots, v_{n}\right\}$. Phrased this way we have the enumeration version of the subset sum problem, a variant of the well-studied knapsack problem. This problem admits a pseudo-polynomial time algorithm via dynamic programming - the very algorithm we used in example 2.27 and illustrated in figure 2.1 .

Example 2.31. Neither $M$ nor $M^{T}$ has any hood vectors. The bolded entries are the only asymmetries in $M$.

\begin{tabular}{|llll|ll|lll|llll|}
\hline 1 & 1 & 0 & 0 & $\mathbf{1}$ & $\mathbf{0}$ & 1 & 0 & 1 & 1 & 0 & 1 & 0 \\
1 & 1 & 0 & 0 & $\mathbf{0}$ & $\mathbf{1}$ & 1 & 0 & 1 & 0 & 1 & 0 & 1 \\
0 & 0 & 1 & 1 & $\mathbf{1}$ & $\mathbf{0}$ & 0 & 1 & 1 & 1 & 0 & 1 & 0 \\
0 & 0 & 1 & 1 & $\mathbf{0}$ & $\mathbf{1}$ & 0 & 1 & 1 & 0 & 1 & 0 & 1 \\
\cline { 1 - 5 } $\mathbf{0}$ & $\mathbf{1}$ & $\mathbf{0}$ & $\mathbf{1}$ & 0 & 0 & 0 & 0 & 0 & 1 & 0 & 1 & 0 \\
$\mathbf{1}$ & $\mathbf{0}$ & $\mathbf{1}$ & $\mathbf{0}$ & 0 & 0 & 0 & 0 & 0 & 0 & 1 & 0 & 1 \\
\hline 1 & 1 & 0 & 0 & 0 & 0 & 0 & 0 & 0 & 1 & 1 & 0 & 0 \\
0 & 0 & 1 & 1 & 0 & 0 & 0 & 0 & 0 & 0 & 0 & 1 & 1 \\
1 & 1 & 1 & 1 & 0 & 0 & 0 & 0 & 0 & 1 & 1 & 1 & 1 \\
\hline 1 & 0 & 1 & 0 & 1 & 0 & 1 & 0 & 1 & 0 & 0 & 1 & 1 \\
0 & 1 & 0 & 1 & 0 & 1 & 1 & 0 & 1 & 0 & 0 & 1 & 1 \\
1 & 0 & 1 & 0 & 1 & 0 & 0 & 1 & 1 & 1 & 1 & 0 & 0 \\
0 & 1 & 0 & 1 & 0 & 1 & 0 & 1 & 1 & 1 & 1 & 0 & 0 \\
\hline$a$ & $b$ & $c$ & $d$ & $e$ & $f$ & $g$ & $h$ & $i$ & $j$ & $k$ & $l$ & $m$
\end{tabular}

Proof. In reduced row echelon form $M$ is 


$$
\left[\begin{array}{rrrrrrrrrrrrr}
a & b & c & d & e & f & g & h & i & j & k & l & m \\
1 & 0 & 0 & -1 & 0 & 0 & 0 & 0 & 0 & 0 & 1 & -1 & 0 \\
0 & 1 & 0 & 1 & 0 & 0 & 0 & 0 & 0 & 1 & 0 & 1 & 0 \\
0 & 0 & 1 & 1 & 0 & 0 & 0 & 0 & 0 & 0 & 0 & 1 & 1 \\
0 & 0 & 0 & 0 & 1 & 0 & 0 & 1 & 1 & 1 & 0 & 0 & -1 \\
0 & 0 & 0 & 0 & 0 & 1 & 0 & 1 & 1 & 0 & 1 & -1 & 0 \\
0 & 0 & 0 & 0 & 0 & 0 & 1 & -1 & 0 & -1 & -1 & 1 & 1 \\
0 & 0 & 0 & 0 & 0 & 0 & 0 & 0 & 0 & 0 & 0 & 0 & 0 \\
0 & 0 & 0 & 0 & 0 & 0 & 0 & 0 & 0 & 0 & 0 & 0 & 0 \\
0 & 0 & 0 & 0 & 0 & 0 & 0 & 0 & 0 & 0 & 0 & 0 & 0 \\
0 & 0 & 0 & 0 & 0 & 0 & 0 & 0 & 0 & 0 & 0 & 0 & 0 \\
0 & 0 & 0 & 0 & 0 & 0 & 0 & 0 & 0 & 0 & 0 & 0 & 0 \\
0 & 0 & 0 & 0 & 0 & 0 & 0 & 0 & 0 & 0 & 0 & 0 & 0 \\
0 & 0 & 0 & 0 & 0 & 0 & 0 & 0 & 0 & 0 & 0 & 0 & 0
\end{array}\right]
$$

This corresponds to

$$
\begin{array}{ll}
a=d-k+l & e=-h-i-j+m \\
b={ }^{-} d-j-l & f=-h-i-k+l \\
c={ }^{-} d-l-m & g=h+j+k-l-m
\end{array}
$$

Where $d, h, i, j, k, l, m$ are independent variables. By choosing

$$
\begin{array}{lll}
d=3^{0}=1 & j=3^{3}=27 & m=3^{6}=729 \\
h=3^{1}=3 & k=3^{4}=81 & \\
i=3^{2}=9 & l=3^{5}=243
\end{array}
$$

We obtain the monopole

$$
\begin{array}{ccccccccccccc}
a & b & c & d & e & f & g & h & i & j & k & l & m \\
\langle 163, & -271, & -973, & 1, & 690, & 150, & -861, & 3, & 9, & 27, & 81, & 243, & 729\rangle
\end{array}
$$

Verifying that there are exactly thirteen $\{0,1\}$-vectors orthogonal to this monopole is left as an exercise to the reader.

The matrices in example 2.27 and example 2.31 both come from a larger family of matrices without hood vectors. 
Example 2.32. Neither $M_{k}$ nor $M_{k}^{T}$ has any hood vectors.

Let $D_{k}$ be the $2 k \times 2 k$ matrix with $2 \times 2$ blocks of $1 \mathrm{~s}$ along the main diagonal and 0 elsewhere. For example,

$$
D_{3}=\left[\begin{array}{llllll}
1 & 1 & 0 & 0 & 0 & 0 \\
1 & 1 & 0 & 0 & 0 & 0 \\
0 & 0 & 1 & 1 & 0 & 0 \\
0 & 0 & 1 & 1 & 0 & 0 \\
0 & 0 & 0 & 0 & 1 & 1 \\
0 & 0 & 0 & 0 & 1 & 1
\end{array}\right]
$$

Let $A_{k}$ be the $2 k \times 2^{k}-1$ matrix whose columns are indicator vectors for a nonempty subset of $[k]$ with each entry repeated twice. For example,

$$
A_{3}=\left[\begin{array}{ccccccc}
\{1\} & \{2\} & \{3\} & \{1,2\} & \{1,3\} & \{2,3\} & \{1,2,3\} \\
1 & 0 & 0 & 1 & 1 & 0 & 1 \\
1 & 0 & 0 & 1 & 1 & 0 & 1 \\
0 & 1 & 0 & 1 & 0 & 1 & 1 \\
0 & 1 & 0 & 1 & 0 & 1 & 1 \\
0 & 0 & 1 & 0 & 1 & 1 & 1 \\
0 & 0 & 1 & 0 & 1 & 1 & 1
\end{array}\right]
$$

Let $01_{m \times n}$ be the $m \times n$ matrix whose entries alternate 0 and 1 in both row and column and with 0 in the upper-left entry. Let $10_{m \times n}$ be the same except with 1 in the upper-left entry. For example,

$$
01_{3 \times 4}=\left[\begin{array}{cccc}
0 & 1 & 0 & 1 \\
1 & 0 & 1 & 0 \\
0 & 1 & 0 & 1
\end{array}\right]
$$

Recall that $J$ is the all-ones matrix. For a given $k$,

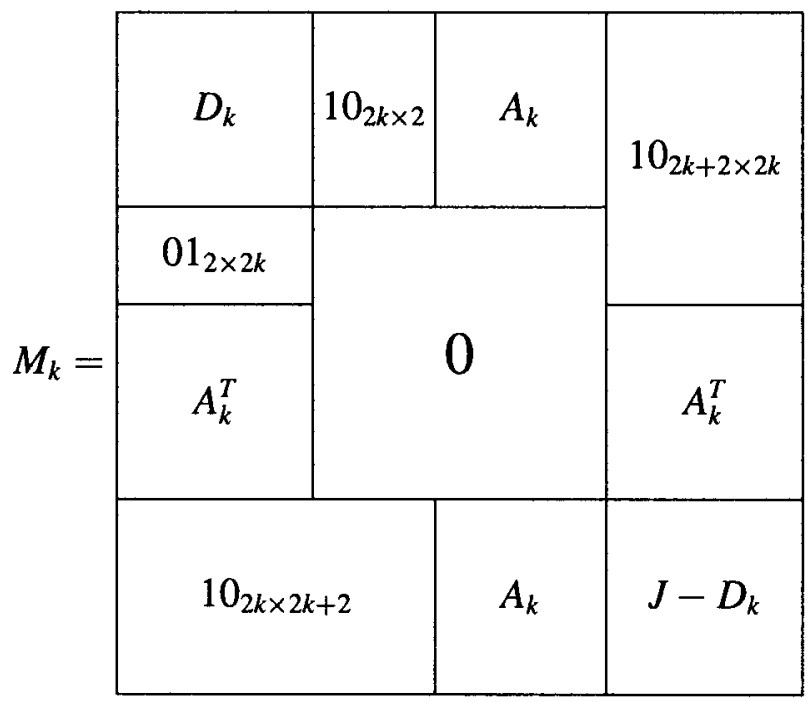


Example 2.33. For each member of the family described in example 2.32, the matrix obtained by replacing the upper-left and lower-right blocks with $\frac{1}{2} J$ and swapping columns $2 k+1$ and $2 k+2$ yields a symmetric matrix with no hood vectors.

\section{5 - Conclusion}

Thus far we have been unable to transform the families of matrices described in example 2.32 and example 2.33 into a counterexample to Conjecture 2.23. However we believe that Conjecture 2.23 is false and that some variation of these families should provide the counterexample, and possibly even a counterexample to Conjecture 2.7. If Conjecture 2.7 or even Conjecture 2.23 is true, example 2.32 and example 2.33 demonstrate that it is true by a very narrow margin. Coupled with Costello and Vu's result [5] about random graphs, it is understandable that this problem is so resistant to attack.

Monopoles provide an interesting side channel for attack. Given a monopole, we can count the number of $\{0,1\}$-vectors orthogonal to the monopole using the dynamic programming approach. In practice this algorithm can test hundreds of millions of monopoles per second on a desktop computer. With enough information about the structure of the monopoles of a potential counterexample we may be able to reduce the search space to a feasible size for a brute-force search. Proposition 2.29 and Proposition 2.30 are first steps in this direction. If a monopole has very few negative (or symmetrically, very few positive) entries then the search algorithm can be made even more efficient by limiting the growth of the dynamic programming table. Proposition 2.30 showed us that we cannot hope to have just a single negative entry, but will two suffice? Or perhaps three? In general a constant bound is unlikely, but perhaps there is a reasonable bound if we restrict ourselves to graphs of order at most $n$. 


\section{CHAPTER 3}

\section{QUERYING: YAO'S PROBLEM}

Many fundamental problems in computer science involve storage and retrieval of information. Given tables of data, for instance the names of all the employees of a business, a storage scheme is devised to record the data in some manner. The retrieval aspect may be concerned with whether a certain entry is present in the table; call this question the membership question. Some algorithm is invoked to inspect the data and return a yes or no answer to the membership question.

The naïve approach to answering the membership question is to inspect, or query, every cell of the table to see if it contains the data in question. If the table has $n$ cells and we ask the membership question for an element that is not in the table, then this algorithm will require $n$ queries to decide the membership question. But remember, we also have control over how data is stored. Suppose the table is sorted upon storage. Now a binary search can answer the membership question in at most $\lceil\lg (n+1)\rceil$ queries. The extra time required to sort the table will be easily eclipsed by the time saved over multiple searches.

\section{1 - Introduction}

In 1981, Andrew Yao asked, ‘Should tables be sorted?' [16]. In particular, he wondered if some restriction on the data could allow us to answer the membership question in fewer queries than the traditional sort/search described above. ${ }^{1}$ He supposed that the table contained $n$ elements chosen from a set of $m$ elements (called the key space and denoted $M$ ), rather than from a potentially infinite set. Let $f(n, m)$ be the number of queries needed in the worst case to answer this membership question. He showed that if the key space is signifi-

\footnotetext{
${ }^{1}$ Binary search on a sorted table is not uniquely the fastest approach. There are other approaches, notably hashing or interpolation search that will perform better for certain applications but the worst-case scenarios still require a number of queries that is at least logarithmic in $n$.
} 
cantly larger than the table then the traditional approach is optimal. That is,

$$
m \gg n \Longrightarrow f(n, m)=\lceil\lg (n+1)\rceil
$$

But when $m$ and $n$ are relatively close in size another store/query strategy can yield improved performance.

He then looked at the question from a different angle: for a fixed $k$ and fixed $n$, what is the largest $m$ such that we can answer the membership question using at most $k$ queries? Let $g(n, k)$ be the largest $m$ such that $f(n, m)=k$.

To obtain a simple lower bound, consider the case when $m=n+1$; i.e., the table contains all but one element of the key space. Picture the key space as a sorted list wrapping around at the ends. Choose the element that is the successor to the missing element and place it in the first cell in the table. Now we can answer the membership question in one query by querying the first cell in the table. That will tell us which element is missing and by extension, which elements are present. Thus $g(n, 1) \geq n+1$.

However this simple approach does not fully take advantage of the tools at our disposal; we just blindly query the first entry of the table. More sophisticated techniques will yield improvements. Yao answered this question for $k=1$ and so determined $g(n, 1)$.

Theorem 3.1 (Yao, [16]).

$$
g(n, 1)= \begin{cases}3 & \text { if } n=2 \\ 2 n-2 & \text { if } n>2\end{cases}
$$

Proof. We will establish that for $n>2, g(n, 1) \geq 2 n-2$ by providing a store/query strategy. Proving that $2 n-2$ is best possible takes up several pages in [16] so the argument is omitted, but Theorem 3.2 will establish that $2 n$ is impossible.

Let $m=2 n-2$. To establish a labeling scheme, suppose that we could force more than two keys to fit into a cell of the table. We could then fit the entire key space into the table by placing two keys in each of the first $n-2$ cells, and one key in cells $n-1$ and $n$. Label the keys in cell $i$ by choosing one to be $K_{i}^{L}$ (the 'lower key') and the other to be $K_{i}^{U}$ (the 'upper 
key'). Treat the lone keys in cells $n-1$ and $n$ as lower keys; these cells have no upper key. This gives our labeling for the key space: $M=\left\{K_{1}^{L}, \ldots, K_{n}^{L}, K_{1}^{U}, \ldots, K_{n-2}^{U}\right\}$.

\begin{tabular}{|c|c|c|c|c|c|}
\hline$K_{1}^{U}$ & $K_{2}^{U}$ & & $K_{n-2}^{U}$ & & \\
\hline$K_{1}^{L}$ & $K_{2}^{L}$ & - • & $K_{n-2}^{L}$ & $K_{n-1}^{L}$ & $K_{n}^{L}$ \\
\hline
\end{tabular}

Given a set $N$ of $n$ keys with which to fill the table, the storage scheme proceeds as follows:

(i) For any cell $i$ such that no key with cell label $i$ is present in $N$, mark the cell as available for other use.

(ii) If the keys with cell label $n-1$ or $n$ are present in $N$ they are added to a list called the shuffle list and the cell is marked as a shuffle cell.

(iii) For the first $n-2$ cells:

(a) If only one of the two keys with that cell label is present in $N$ then that key is stored in the cell.

(b) If both keys with that cell label are present in $N$ then by the pigeonhole principle another cell must be marked as available via (i). The upper key for the current cell is stored in the available cell. The lower key for the current cell is added to the shuffle list. The current cell is marked as a shuffle cell.

(iv) At this stage, every cell marked as available in (i) is filled with an upper key with a different cell label. The other cells are either filled with a key with the corresponding cell label or are marked as a shuffle cell.

(v) The shuffle list must have at least two keys, since by the pigeonhole principle for each of $K_{n-1}^{L}$ and $K_{n}^{L}$ that are not in $N$ there must have been a cell for which both keys with that cell label are present in $N$. Note that every key on the shuffle list is a lower key and that there are the same number of shuffle cells as keys on the shuffle 
list. Complete the table by assigning the keys from the shuffle list to shuffle cells in such a way that no shuffle cell is assigned a key with that cell label (a cyclic shift will suffice).

To answer the membership question for a key with cell label $i$, we query cell $i$.

- If cell $i$ holds $K_{i}^{L}$ or $K_{i}^{U}$ then the key was assigned by (iii.a) and so the other key with that cell label is not in the table.

- If cell $i$ holds $K_{j}^{U}, j \neq i$ then the key was assigned by (iii.b). Therefore cell $i$ was marked as available by (i) and so neither $K_{i}^{L}$ nor $K_{i}^{U}$ is in the table.

- If cell $i$ holds $K_{j}^{L}, j \neq i$ then the key was assigned by (v). Therefore cell $i$ was marked as a shuffle cell by (ii) or by (iii.b) and so both $K_{i}^{L}$ and $K_{i}^{U}($ if $i<n-1)$ are present in the table.

Thus $f(n, 2 n-2)=1$ and so $g(n, 1) \geq 2 n-2$.

Although Yao takes several pages to prove the upper bound half of Theorem 3.1, the start of his argument can be adapted into a quick proof leaving a gap of 1 .

Theorem 3.2. $g(n, 1) \leq 2 n-1$

Proof. Suppose we have a store/search strategy for a key space containing at least $2 n$ keys. By the pigeonhole principle, the search strategies for at least two keys will query the same cell. Without loss of generality, the search for keys 1 and 2 both query cell 1 .

Let $Y_{1}$ (the 'yes set' for key 1) be the set of keys whose appearance in cell 1 resolve the membership question for key 1 in the affirmative. All keys not in $Y_{1}$ are in $N_{1}$, the 'no set' for key 1. Define $Y_{2}$ and $N_{2}$ similarly.

Claim: $\left|Y_{1}\right| \leq n$.

If $\left|Y_{1}\right|>n$, then there is a subset $S$ of $Y_{1}$ of cardinality $n$ that does not contain key 1 . But if the table is populated with the keys from $S$, then the first cell will contain a key that will falsely imply that key 1 is in the table, contradicting that this store/search strategy is valid.

Therefore $\left|N_{1}\right| \geq n$. Since key 1 is clearly in $Y_{1}$, we can populate the table with key 1 and $n-1$ keys chosen from $N_{1}$ without choosing key 2 . The storage scheme must place key 
1 in cell 1 , and so key 1 must belong to $N_{2}$. Similarly, key 2 must belong to $N_{1}$.

Now populate the table using key 1 , key 2 , and $n-2$ keys chosen from $N_{1}$. The storage scheme must place key 1 in cell 1 since all other keys belong to $N_{1}$. But since key 1 is in $N_{2}$, when we attempt to answer the membership question for key 2 we will see key 1 in cell 1 and falsely imply that key 2 is not in the table. Thus, this search/store strategy cannot be valid.

\section{2 - Two queries}

At his 2010 REGS, Doug West proposed [15] that we try to determine the maximum size of the key space when we are permitted two queries.

If the table has only three cells then we can accommodate a key space of any size by storing the keys in the table in sorted order. With the first query we query the middle cell. If that is the key we are looking for then great, we are done. If that key is larger than the key we are looking for then we query the first cell. Otherwise we query the last cell. Therefore $g(3,2)=\infty$ and more generally, using a similar sort/search strategy, $g\left(2^{k}-1, k\right)=\infty$. Thus we only need to consider tables with at least four cells.

Proposition 3.3. For $n \geq 4, g(n, 2) \geq 3 n-4$.

Proof. Start by labeling all the keys in $M$ using a technique similar to Yao's from Theorem 3.1. For $i \in[n-2]$, three keys will have cell label $i$ : a 'lower key' $K_{i}^{L}$, a 'middle key' $K_{i}^{M}$, and an 'upper key' $K_{i}^{U}$. For $i \in\{n-1, n\}$ only one key will have cell label $i: K_{n-1}^{U}$ and $K_{n}^{M}$.

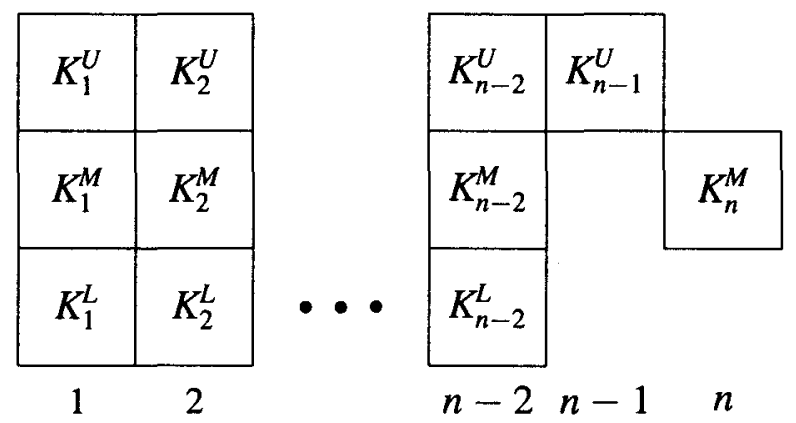


Given a set $N$ of $n$ keys from the key space, the storage scheme works as follows:

(i) Examine the first $n-2$ cells.

(a) If no keys in $N$ have this cell label, then the cell is flagged as available.

(b) If only one key in $N$ has this cell label then that key is assigned to the cell.

(c) If two of more keys in $N$ have this cell label then we classify the cell by type:

\begin{tabular}{|c|c|c|c|}
\hline Type $A$ & Туре $B$ & Type $C$ & Type $D$ \\
\hline$K_{i}^{U}$ & $K_{i}^{U}$ & & $K_{i}^{U}$ \\
\hline$K_{i}^{M}$ & & $K_{i}^{M}$ & $K_{i}^{M}$ \\
\hline$K_{i}^{L}$ & $K_{i}^{L}$ & $K_{i}^{L}$ & \\
\hline
\end{tabular}

(ii) Pair up cells of type $A$ and $B$ with other cells of type $A$ and $B$.
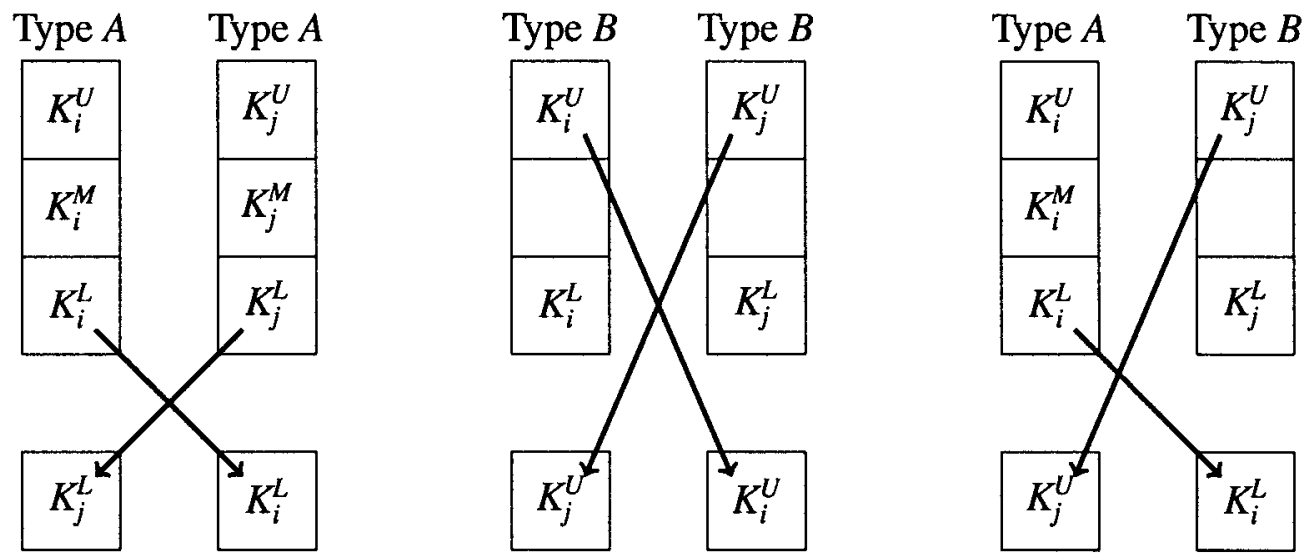

(iii) We may have one extra cell of type either $A$ or $B$. Now consider that extra cell $i$ (if it exists) and cell $n-1$.
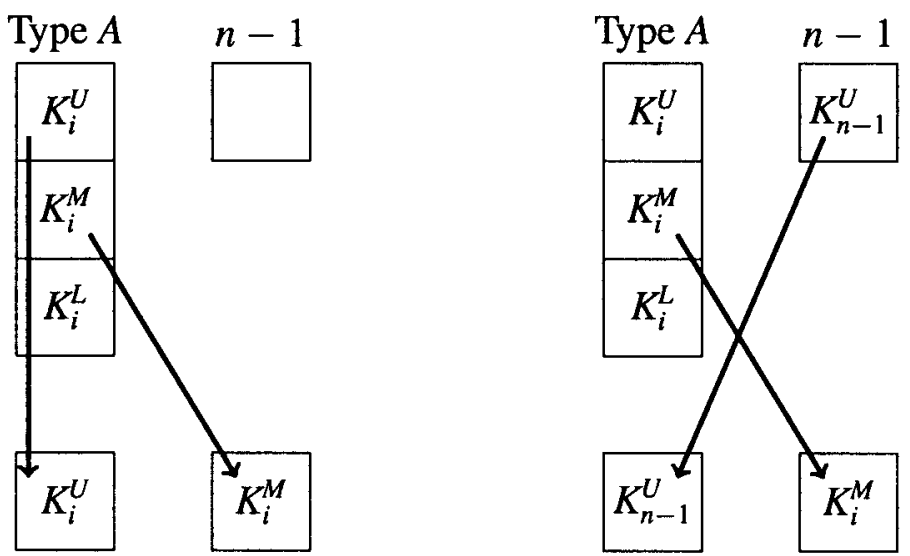

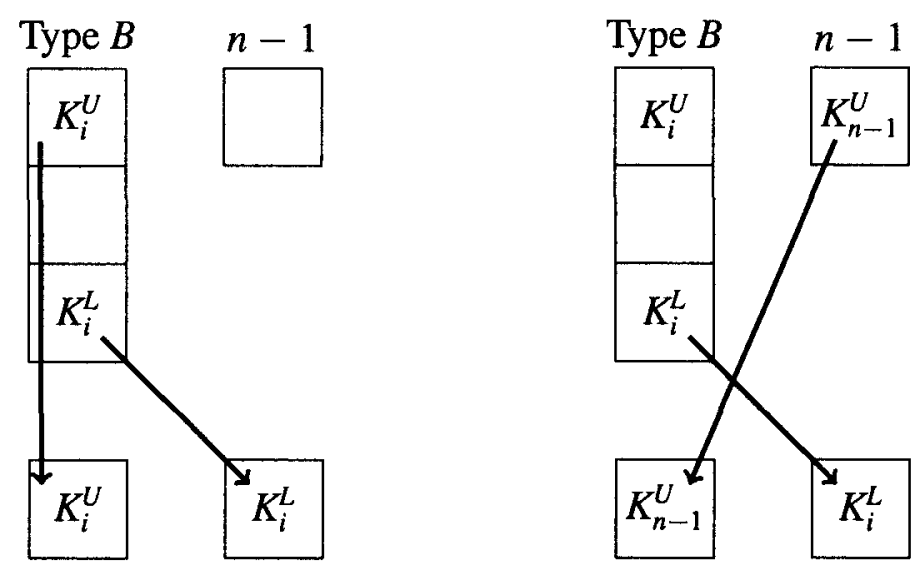

(iv) Pair up cells of type $C$ and $D$ with other cells of type $C$ and $D$.
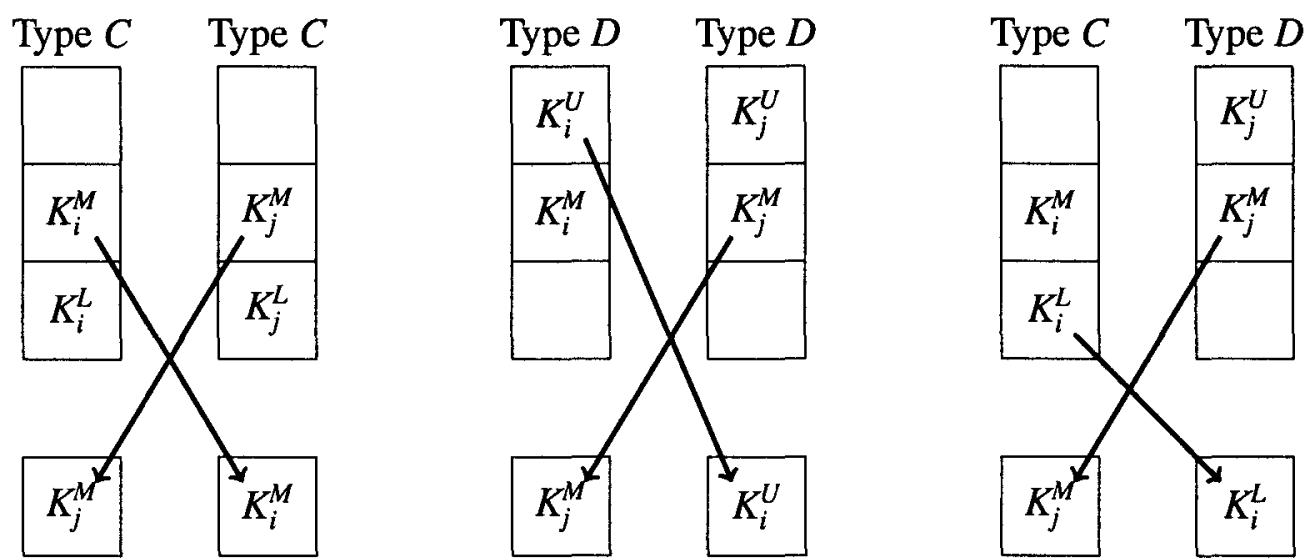

(v) We may have one extra cell of type either $C$ or $D$. Now consider that extra cell $i$ (if it exists) and cell $n$.
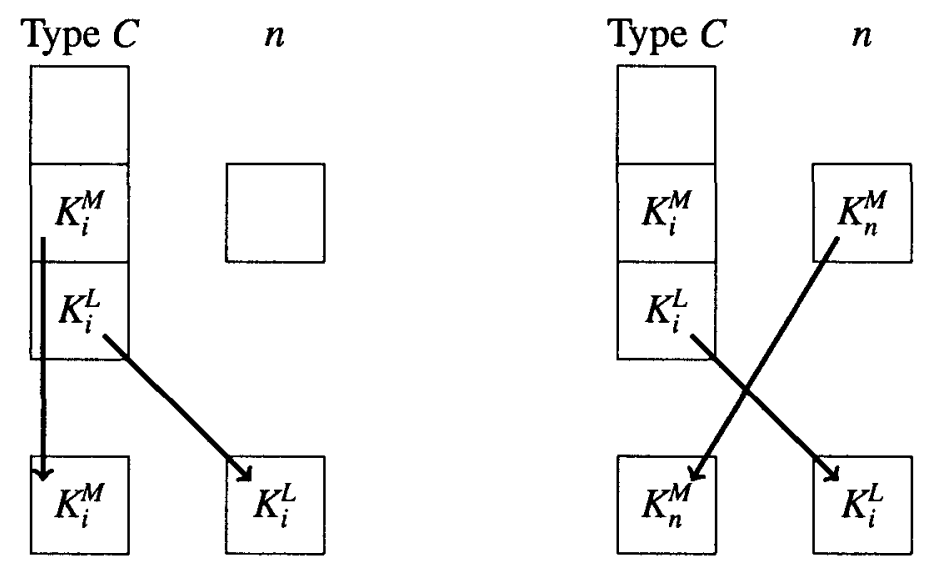


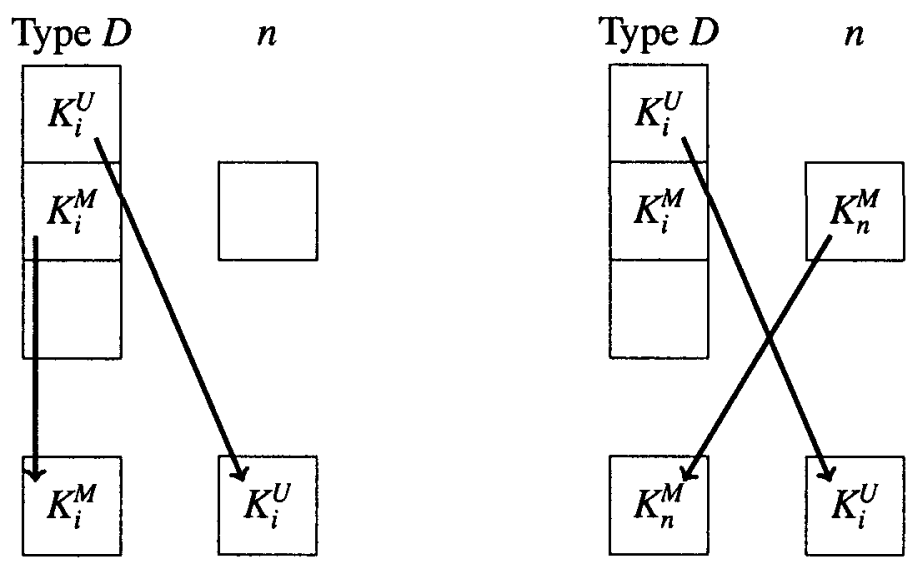

(vi) Fill any cells flagged as available with any remaining keys.

Thus we are able to answer the membership question in at most two queries for a table of $n$ keys chosen from a key space of size $3 n-4$ and so $g(n, 2) \geq 3 n-4$.

When we were permitted only a single query, we had to know in advance which cell we would choose to query when presented with the membership question for a specific key. With two queries this is no longer necessarily the case. While we still need to know the first cell to query, the strategy given in Proposition 3.3 is adaptive: it uses information gained from the first query to decide which other cell to query. We could mandate that the strategy be non-adaptive, meaning that when we are presented with the membership question for a specific key the algorithm decides which cells to query before making any queries to the table. The best-known upper bound for the two query problem was established by David Howard but is an upper bound only for the non-adaptive case.

Proposition 3.4 (Howard, [7]). If restricted to non-adaptive strategies,

$$
g(n, 2) \leq \mathrm{O}\left(n^{2} \log n\right)
$$

Yao's original question was motivated by situations in which the key space was restricted. One approach to the two query problem is to impose further restrictions on the key space. If we can solve that problem, we may be able to solve the original problem by tiling the original key space with copies of the restricted space. 


\section{3 - Graph Queryability}

Yao restricted the key space by supposing that it contained only $m$ keys. However, any subset of size $n$ is permitted. Suppose we further mandate that only certain subsets of the key space are permitted. If we consider subsets of size 2 we can represent the restrictions as a graph with the key space as vertices and with edges between pairs of keys that are permitted to appear together.

Example 3.5. Let $\{a, b, c, d\}$ be the key space and suppose that we permit all pairs except $\{b, d\}$. This can be represented as the diamond graph $K_{4}-e$, where $b d$ is the deleted edge.

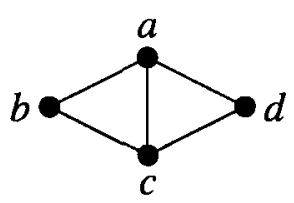

Diamond $K_{4}-e$

Definition 3.6. A graph $G$ is 1-queryable means there exists a one-query sort/search strategy that will answer the membership question for any element of $V(G)$ on any table formed from an edge of $G$.

Example 3.7. The complete graph on three vertices, $K_{3}$, is 1-queryable.

Proof. We will use this example to demonstrate how the sort/search strategy can be encoded into the graph. The table contains two cells. Every permissible subset of size 2 is an edge of the graph. To indicate how a given pair of keys will be stored in the table, we orient the corresponding edge such that the orientation $u \rightarrow v$ indicates that $u$ will be stored in the first cell of the table and $v$ will be stored in the second cell. So if we have the orientation

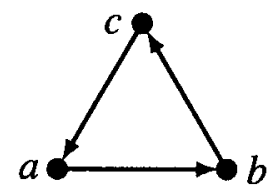

and we are presented with $\{a, c\}$ to store in the table, we will store $c$ in the first cell and $a$ in the second cell.

When we are asked to answer the membership question for a key $v$, we must decide 
which cell of the table to query. Suppose we decide to query the first cell. We can indicate this decision in the graph by coloring vertex $v$ color 0 . So if we have the coloring

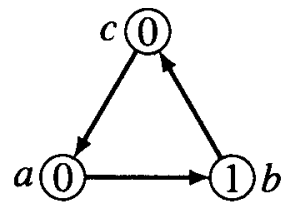

then we will query the first cell when asked the membership question for $a$ or $c$ and we will query the second cell when asked the membership question for $b$.

Under the encoding given, opening either door will determine exactly which edge is stored in the table. Thus $K_{3}$ is 1-queryable and the given encoding is valid.

It is refreshingly easy to characterize valid encodings. Notice that given a valid encoding, if we reverse the orientation of every edge and the color of every vertex then we have another valid encoding. We will use this fact on multiple occasions to fix the color of a vertex without any loss of generality.

Lemma 3.8. An encoding of $G$ is valid if and only if for every edge $u \rightarrow v$ :

(i) if $v$ has color 0 then $d^{+}(u)=1$

(ii) if $u$ has color 1 then $d^{-}(v)=1$
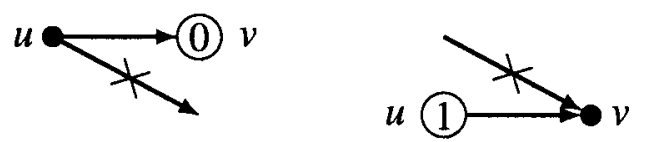

Proof. Suppose every edge satisfies (i) and (ii) and that we are asked to answer the membership question for vertex $a$. Without loss of generality, assume $a$ has color 0 . If we query the first cell and find $a$ then we are done; if not, let $b$ be the vertex found in the first cell. If $a b$ is not an edge of $G$ or if the edge is encoded as $a \rightarrow b$ then we conclude that $a$ is not in the table. Otherwise the edge $a b$ is encoded as $b \rightarrow a$. Since $a$ has color 0 , we know that $d^{+}(b)=1$ by (i). Therefore $a$ must be in the second cell of the table. Thus we can decide the membership question in one query with this strategy and so the encoding is valid.

Now suppose that some oriented edge $c \rightarrow d$ does not satisfy both (i) and (ii). If it does not satisfy (i) then $d$ has color 0 and $d^{+}(c)>1$. When the table contains the edge $c d$ and 
we are asked to solve the membership question for $d$, we will query the first cell and find $c$. But since $d^{+}(c)>1$, there is another vertex $x$ that is oriented $c \rightarrow x$. Thus we are unable to decide the membership question for $d$ in one query using this encoding, and so the encoding is not valid. The argument is similar in the case that (ii) is not satisfied.

If $G$ is a bipartite graph with partite sets $X$ and $Y$, consder the encoding given by coloring all vertices in $X$ color 0 , all vertices in $Y$ color 1 , and orienting all edges to point from $X$ to $Y$. This encoding will vacuously satisfy the requirements of Lemma 3.8 .

Corollary 3.9. Every bipartite graph is 1-queryable.

Lemma 3.10. In a valid encoding of a 1-queryable graph, every triangle must be oriented as a directed cycle.

Proof. Let $a, b$, and $c$ be the vertices of the triangle. Without loss of generality $c$ is colored 0 and the edge $a b$ is oriented $a \rightarrow b$. It follows by Lemma 3.8 that $a c$ must be oriented $c \rightarrow a$. To obtain a contradiction, assume $b c$ is oriented $c \rightarrow b$. Both $a$ and $b$ must be colored 1 by Lemma 3.8. This is a contradiction, since $d^{+}(b) \geq 2$.
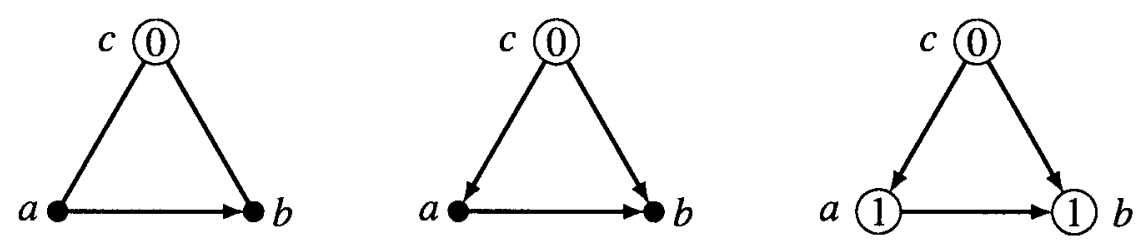

Lemma 3.11 (3-chaining). Let $v_{0}, v_{1}, \ldots, v_{n}$ be a path in a graph $G$ with $d\left(v_{i}\right) \geq 3$ for $i=1, \ldots n$. If $v_{0}$ and $v_{1}$ have color 0 and the edge $v_{0} v_{1}$ is oriented $v_{1} \rightarrow v_{0}$ then:

(i) $v_{1}, \ldots, v_{n}$ all have color 0 .

(ii) The edges $v_{i-1} v_{i}$ are oriented $v_{i} \rightarrow v_{i-1}$ for $i=1, \ldots, n$.

(iii) Every neighbor $u$ of $v_{i}$ has color 0 and $u v_{i}$ is oriented $u \rightarrow v_{i}$ (except when $u=v_{i-1}$ ) for $i=1, \ldots, n$.

(iv) For any $1 \leq i<j \leq n$, if $j \neq i+1$ then $v_{i}$ is not adjacent to $v_{j}$.

Proof. The hypotheses, together with Lemma 3.8 imply that $d^{+}\left(v_{1}\right)=1$, and so all edges incident to $v_{1}$, aside from $v_{0} v_{1}$, must flow into $v_{1}$. Thus $d^{-}\left(v_{1}\right) \geq 2$, so all vertices adjacent to $v_{1}$ must have color 0 . This provides the base case for the claim, and the rest follows by 
induction on $n$.
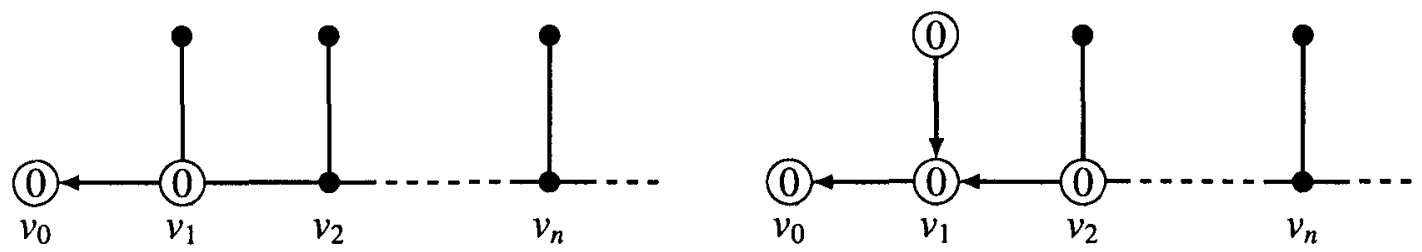

Corollary 3.12. Let $C$ be an odd cycle in a graph $G$ such that every vertex of $C$ has degree at least 3 .

- $C$ must be oriented as a directed cycle and must be monochromatic.

- Any vertex in $G-C$ that is adjacent to a vertex in $C$ must also have that same color.

- $C$ acts as either a source or a sink for the vertices of $G-C$ that are adjacent to a vertex of $C$, according to whether the color is a 0 or a 1 , respectively.

Lemma 3.13. Let $G$ be a 1-queryable graph with no vertices of degree two. Either $G$ is bipartite, or $G$ is unicyclic.

Proof. Suppose $G$ is neither bipartite nor unicyclic. $G$ has an odd cycle $C$ with minimum degree three, so by Corollary $3.12, C$ is monochromatic. Without loss of generality, $C$ has color 0 and is a sink. $C$ cannot have a chord, as a chord would not be orientable.

If another cycle shares two vertices with $C$ then there is a path between two vertices $u$ and $v$ of $C$. Call the interior vertices of the path $p_{1}, p_{2}, \ldots, p_{k}$. Starting at $v$ and applying Lemma 3.11 , every $p_{i}$ must be colored 0 , and all edges must be oriented $p_{i} \rightarrow p_{i+1}$. But now $d^{+}\left(p_{1}\right)=2$, contradicting Lemma 3.8 .

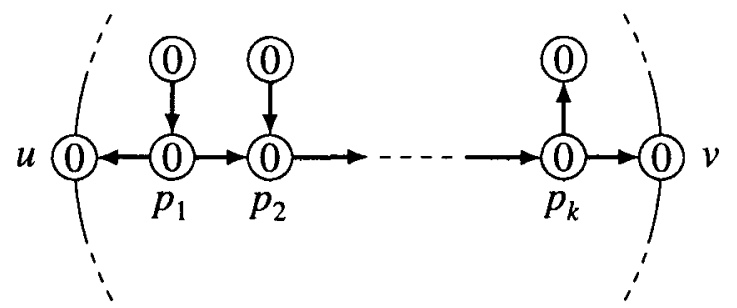

Thus any other cycle of $G$ must be connected to $C$ by a path. This is a contradiction to Lemma 3.11, completing the proof.

Lemma 3.14. Let $G$ be a 1-queryable graph and let $S$ be the set of all vertices of $G$ of degree 
two. Every component of $G-S$ is either bipartite or unicyclic.

Proof. Suppose $G-S$ has a component that is neither bipartite nor unicyclic. This component has at least one odd cycle, and since we removed all vertices of $G$ of degree two, every vertex of this odd cycle has minimum degree at least three. Thus, by Corollary 3.12 it is monochromatic. The component also contains another cycle, which either shares vertices with the odd cycle or is connected to the cycle by a path. Either way, in $G$ all the relevant vertices have minimum degree three, and so by Lemma $3.13, G$ has a subgraph that is not 1-queryable, contradicting that $G$ is 1-queryable.

Theorem 3.15. Every 1-queryable graph has chromatic number at most three.

Proof. Let $G$ be a 1-queryable graph and let $S$ be the set of all vertices of $G$ of degree two. By Lemma 3.14, every component of $G-S$ is either bipartite or unicyclic and so can be colored with at most three colors. Hence $G-S$ has a valid coloring using colors chosen from the set $\{a, b, c\}$. This coloring can be extended to a valid coloring of $G$ by coloring each vertex $v$ of $S$ in turn. Since every vertex in $S$ has degree two, at least one of the colors from $\{a, b, c\}$ is unused by the neighbors of $v$. Thus the coloring can be extended to $v$ and hence to all of $G$.

\section{4 - Conclusion}

This chapter studied a storage problem introduced by Yao and resurrected by Doug West in his 2010 REGS [15]. Section 3.2 gives a lower bound for the 2-query, adaptive version of this problem. No known upper bound even comes close to matching this lower bound, so section 3.3 revisits the structure of extremal 1-queryable graphs in the hopes that this will provide a tiling argument leading to a better upper bound for the 2-query case. While this section does give a near-complete characterization of 1-queryable graphs, a generalization to 1-queryable $k$-uniform hypergraphs is required to power this tiling which reduces the

2-queryable problem to a 1-queryable problem with an open cell. An 'open cell' whose contents are always known. With one query and one open cell it is possible to accommodate 
a key space of $2 n-1$ keys, one more than Yao's bound for the basic 1-query case; we conjecture that this is the best possible. Another variant is to allow for 'closed' cells: ones that cannot be queried. The bounds for this problem are very close to the bounds for the original problem.

There has been some progress on characterizing 1-queryable 3-uniform hypergraphs. If for every pair of vertices the co-degree 0 or at least 7 then the hypergraph must be tripartite. This is the 3-uniform analogue to the fact that if a 1-queryable graph has minimum degree at least 3 then the graph must be bipartite. A complete characterization of 1-queryable graphs is not yet known, nor is a generalization to graphs of higher uniformity. The co-degree restriction imposes a sort of edge-density requirement on the hypergraph. It remains an open problem to determine the maximum number of edges that can be contained in a $k$ uniform 1-queryable hypergraph, but it is known that the extremal hypergraphs will not be $k$-partite. 


\section{REFERENCES}

[1] S. Akbari, P. Cameron, and G. Khosrovshahi, "The rank and signature of adjacency matrices," in preparation, 2004.

[2] C. Berge, Hypergraphs. Amsterdam: North-Holland Publishing Co., 1989, pp. x +255 , ISBN: 0-444-87489-5.

[3] T. C. Brown, P. Erdôs, and A. R. Freedman, "Quasi-progressions and descending waves," J. Combin. Theory Ser. A, vol. 53, no. 1, pp. 81-95, 1990, ISSN: 0097-3165. DOI: $10.1016 / 0097-3165(90) 90021-N$.

[4] P. J. Cameron, "Research problems from the BCC21," Discrete Math., vol. 310, no. 2, pp. 347-354, 2010, ISSN: 0012-365X. DOI: 10.1016/j.disc.2009.04.016.

[5] K. P. Costello and V. H. Vu, "The rank of random graphs," Random Structures Algorithms, vol. 33, no. 3, pp. 269-285, 2008, ISSN: 1042-9832. DOI: 10.1002/rsa. 20 219.

[6] R. Graham, "Some of my favorite problems in Ramsey theory," in Combinatorial number theory, Berlin: de Gruyter, 2007, pp. 229-236.

[7] D. Howard, personal communication, 2010.

[8] B. M. Landman, "Ramsey functions for quasi-progressions," Graphs Combin., vol. 14, no. 2 , pp. 131-142, 1998, ISSN: 0911-0119. DOI: $10.1007 / \mathrm{s} 003730050021$.

[9] B. M. Landman and A. Robertson, Ramsey Theory on the Integers. American Mathematical Society, 2004, pp. xvi+317, ISBN: 0-8218-3199-2.

[10] D. C. Lay, Linear Algebra and its Applications, second ed. update. Addison-Wesley Publishing Co., 2000, pp. xx+555, ISBN: 0-201-34771-1. 
[11] R. Raz and B. Spieker, "On the "log rank"-conjecture in communication complexity," Combinatorica, vol. 15 , no. 4 , pp. 567-588, 1995, ISSN: 0209-9683. DOI: 10.100 7/BF01192528.

[12] A. C. M. van Rooij and H. S. Wilf, "The interchange graph of a finite graph," Acta Math. Acad. Sci. Hungar., vol. 16, pp. 263-269, 1965, ISSN: 0001-5954.

[13] S. Vijay, "On a variant of van der waerden's theorem," Integers, vol. 10, A17, 223227, 2010, ISSN: 1867-0652. DOI: 10.1515/INTEG.2010.017.

[14] D. B. West, Introduction to Graph Theory, second ed. Prentice Hall Inc., 2001, pp. $x i x+588$, ISBN: 0-13-014400-2.

[15] —, (2010). Yao's table storage problem, URL: http : / / www . math . uiuc . edu / -west/regs/yao.html.

[16] A. C. C. Yao, "Should tables be sorted?"' J. Assoc. Comput. Mach., vol. 28, no. 3, pp. 615-628, 1981, ISSN: 0004-5411. DOI: 10.1145/322261.322274. 


\section{GLOSSARY OF NOTATION}

Aut $G$ automorphism group of $G$

$\chi(G) \quad$ chromatic number of $G$

$d_{G}(u, v), d(u, v) \quad$ distance from $u$ to $v$ in $G$

$d_{G}(v), d(v) \quad$ the degree of $v$ in $G$

$d_{G}^{+}(v), d^{+}(v) \quad$ the outdegree of $v$ in $G$

$d_{G}^{-}(v), d^{-}(v)$ the indegree of $v$ in $G$

$\Delta(G)$ the maximum degree of $G$

$\delta(G)$ the minimum degree of $G$

$\operatorname{diam} G \quad$ diameter of $G$

$\gamma(G)$ domination number of $G$

$\gamma_{t}(G)$ total domination number of $G$

$K_{n} \quad$ complete graph on $n$ vertices

$K_{m, n} \quad$ complete bipartite graph

$N_{G}(v), N(v)$ the (open) neighborhood of $v$ in $G$

$N_{G}[v], N[v]$ the closed neighborhood of $v$ in $G$

$\omega(G)$ clique number of $G$

$P_{n} \quad$ path on $n$ vertices 


\section{INDEX}

adjacency matrix, 20

block, 4

major, 4

minor, 4

claw graph $\left(K_{1,3}\right), 24$

diameter

of a quasi-progression, 1

diamond graph $\left(K_{4}-e\right), 24,51$

even triangle, 24

$f(n, m), 43$

$g(n, k), 44$

graph

1-queryable, 51

valid encoding, 52

adjacency matrix, 20

rank, 20

reduced, 21

hood vector, 22

line graph, 24

lollipop graph $\left(L_{3,1}\right), 23$ low-difference, 1,3

major block, 4

major superblock, 5

membership question, 43

minor block, 4

minor superblock, 5

monopole, 31, 33-34

neighborhood

shadow, 22

vector, 22

neighborhood vector, 22

odd triangle, 24

$Q(d, k), 1,3$

Quasi-progression, 1

query, 43

rank

of a graph, 20

reduced graph, 21

shadow neighborhood, 22

superblock, 5

extremes, 5 
major, 5

minor, 5

triangle

even, 24

odd, 24

twin, 21

valid encoding, 52

van der Waerden number, 1

vertex

twin, 21 


\section{CURRICULUM VITE}

Adam S. Jobson

\section{Education}

2011 University of Louisville Ph.D. in Applied and Industrial Mathematics

2006

\section{University of Louisville}

M.S. in Mathematics

2004

\section{University of Louisville}

B.S. in Mathematics

\section{Publications}

The Hub Number of a Graph (with T. Grauman, S. G. Hartke, B. Kinnersley, D. B. West, L. Wiglesworth, P. Worah, H. Wu), Inform. Process. Lett. 108 (2008), 226-228

On Hamiltonian colorings and hc-stable graphs (with B. Allgeier, G. Chartrand, L. Nebeský, P. Zhang), Proceedings of the Thirty-Ninth Southeastern International Conference on Combinatorics, Graph Theory and Computing (2008) 65-76

Stack Domination Density (with T. Brauch, P. Horn, D. J. Wildstrom), submitted.

Ramsey Functions for Quasi-Progressions with Large Diameter (with A. Kézdy, H. Snevily, S. White), submitted. 